\title{
Development and Validation of a Robust Ferroptosis-Related Prognostic Signature to Predict Overall Survival in Clear Cell Renal Cell Carcinoma
}

\section{Yen-ting Lin}

The First Affiliated Hospital of Jinan University

Can-Xuan Li ( $\nabla$ china20170713@163.com )

The First Affiliated Hospital of Jinan University

Jie Chen

The First Affiliated Hospital of Jinan University

\section{Research Article}

Keywords: Ferroptosis, clear cell renal cell carcinoma, prognostic model, TCGA

Posted Date: February 25th, 2021

DOI: https://doi.org/10.21203/rs.3.rs-230598/v1

License: (c) (i) This work is licensed under a Creative Commons Attribution 4.0 International License.

Read Full License 


\section{Abstract}

Background: Ferroptosis is a novel defined type of programmed cell death (PCD) with widespread functions involved in physical conditions or multiple diseases including malignancies. However, the relationship between ccRCC and ferroptosis-related regulators remains poorly known. Herein, we investigate the prognostic values and potential mechanisms of ferroptosis-related genes (FRGs) in cCRCC.

Methods: Ferroptosis-related genes were obtained from FerrDb database, GeneCards database and previously published literatures. The gene expression profile of ferroptosis-related regulators and corresponding clinicopathological information were downloaded from The Cancer Genome Atlas (TCGA). Differentially expressed ferroptosis-related genes (DE-FRGs) were screened between ccRCC specimens and noncancerous specimens. Among these genes, prognostic DE-FRGs were identified using univariate COX analysis and LASSO regression analysis. Further multivariate COX regression was employed to identify prognosis-related hub DE-FRGs and establish a prognostic model.

Results: We identified seven hub genes (HMGCR, MT1G, BID, EIF4A1, FOXM1, TFAP2C and CHAC1) from the DE-FRGs using univariate Cox regression analysis, LASSO and multivariate Cox regression analysis, and used them to establish a novel clinical predictive model in the TCGA train cohort $(n=374)$. Subsequently, we assessed the prognostic value of the model. Survival analysis showed that high-risk patients had a reduced overall survival (OS), the time-dependent receiver operating characteristic (ROC) curve analysis confirmed the signature's diagnostic performance. Additionally, multivariate Cox regression analysis suggested that the risk score was an independent prognostic factor. Additionally, we verified the prognostic performance of the risk model in the testing cohort $(n=156)$, and the entire group $(n=530)$ using Kaplan-Meier curve and ROC curve analyses. Functional analysis indicated that several carcinogenic pathways were enriched, and tumor-infiltrating immune cell abundances, and the expression levels of immunosuppressive molecules were different between two risk groups. Finally, external databases (ONCMINE, GEPIA, HPA, Kaplan-Meier plotter and cbioportal) were used to confirm the expression patterns, prognostic value, and genetic mutations of 7 hub FRGs in ccRCC.

Conclusions: Collectively, we successfully constructed a novel ferroptosis-related risk signature that was significantly associated with the prognosis of ccRCC.

\section{Introduction}

Renal cell carcinoma (RCC) is one of the most lethal types of urogenital malignancies, accounting for approximately $2-3 \%$ of malignancies ${ }^{[1]}$. Thereinto, clear cell renal cell carcinoma is the most common pathological type of RCC, which exhibits high mortality and poor prognosis ${ }^{[2]}$. Surgical resection is the most ideal option for localized ccRCC, however, around $20 \%-40 \%$ of postoperative patients experience recurrence or metastasis ${ }^{[3,4]}$. Recurrence and metastasis are the major reasons of ccRCC-related mortality ${ }^{[5]}$. Although the development of treatment, the prognosis of this disease still poor, especially for 
advanced metastasis patients ${ }^{[6-8]}$. Hence, it is significantly urgent to search for more sensitive therapeutic biomarkers and investigate its precise mechanisms

Ferroptosis, a novel discovered form of regulated cell death, is different from apoptosis, cell necrosis, and autophagy $[9,10]$. It is defined as an oxidative, iron-dependent form of programmed cell death that is usually accompanied by the accumulation of reactive oxygen species (ROS) and lipid peroxides ${ }^{[11]}$. The system $\mathrm{XC}^{-}$inhibition and glutathione peroxidase 4 (GPX4) inactivation are the two crucial mechanisms of ferroptosis activation ${ }^{[12,13]}$. System ${X C^{-}}^{-}$simultaneously transports cystine into the cells and exports glutamate outside the cells. The system $\mathrm{XC}^{-}$inhibition reduces the intracellular cysteine (the precursor for the synthesis of GSH). GSH plays a vital role in cellular antioxidant defenses. GSH depletion leads to the iron-dependent accumulation of ROS and subsequent ferroptosis. GPX4 plays a crucial role in preventing ferroptosis, GPX4 uses GSH as a co-factor for catalyzing peroxides into lipid alcohols, thus limiting the accumulation of lipid peroxides. Preclinical trials confirmed that knocking out GPX4 resulted in embryonic lethality and accumulation of large amounts of lipid peroxides ${ }^{[14]}$.

In recent years, there has been a great deal of effort to investigate the molecular mechanisms of ferroptosis in cancer development ${ }^{[15]}$, emerging evidence showed that triggering ferroptosis could inhibit tumor progression, and inhibiting ferroptosis has the opposite effect ${ }^{[16]}$. The roles of ferroptosis in tumor treatment has gradually been discovered. Sorafenib could induce ferroptosis of hepatocellular carcinoma cells by inhibiting system $\mathrm{xc}$ and depleting $\mathrm{GSH}^{[17]}$. Additionally, Lapatinib could cause ferroptosis of breast cancer cells when it is used with siramesine ${ }^{[18]}$. Targeting ferroptosis-related regulators is a promising strategy for cancer therapy. However, only a few studies have in-depth investigated the ferroptosis-related genes and pathways in ccRCC. During the initial and progression of CCRCC, the detailed signal transduction mechanisms and vital regulatory factors of ferroptosis remains largely unknown.

Herein, we systematically investigated the characteristics of ferroptosis-related genes in ccRCC based on data from the TCGA database, Figure 1 showed the workflow of this study. In conclusion, we constructed a predictive model based on ferroptosis-related genes to assist clinicians in prognostic stratification and clinical decisions of ccRCC patients.

\section{Materials And Methods}

\section{Data Source}

The RNA-sequencing data of 539 ccRCC samples and 72 normal renal samples, normalized by fragments per kilobase of transcript per million mapped reads (FPKM), was downloaded from TCGA database (https://portal.gdc.cancer.gov/). Duplicate specimens and any samples with unavailable or unknown survival time data will be excluded, and finally we obtained RNA sequencing profile of 72 normal renal specimens and $530 \mathrm{ccRCC}$ specimens. Meanwhile, corresponding clinicopathological variables of 530 
patients with ccRCC, including age, gender, clinical stage, histological grade, tumor size (T status), distant metastasis (M status), and lymph node metastasis ( $\mathrm{N}$ status) were obtained and listed in Table 1.

\section{Differentially expression ferroptosis-related genes (DE-FRGs) and functional enrichment analysis}

The "limma" package built in R was used to identify the differentially expressed ferroptosis-related genes (DE-FRGs) between ccRCC specimens and normal control specimens. Adjusted $\mathrm{P}<0.05$ and llog2fold change (FC) $\mid \geq 1$ was considered statistically significant. Gene Ontology (GO) and the Kyoto Encyclopedia of Genes and Genomes (KEGG) analyses of DE-FRGs were explored using the "clusterProfiler" R package. All functional enrichment items with the false discovery rate (FDR) $\leq 0.05$ were considered significant.

\section{Development and validation of a prognostic ferroptosis-related gene signature}

Firstly, we performed univariate Cox analysis to identify DE-FRGs associated with overall survival, and genes with the cut-off value of $p<0.05$ were considered significant. Secondly, using the "glmnet" $R$ package, LASSO regression analysis was used to minimize the variables and chose the most significant prognosis-associated DE-FRGs. Thirdly, all Patients in TCGA dataset was randomly divided into training set and test set according to the ratio of 7:3. Subsequently, we conducted stepwise multivariate Cox regression analysis in the training cohort to identify prognosis-related hub FRGs and construct the prognostic signature. Afterwards, risk score of each ccRCC patients was computed with the following formula: exp gene $1 * \beta$ gene $1+\exp$ gene $2 * \beta$ gene $2+\exp$ gene $3 * \beta$ gene $3+\ldots$ exp gene $n * \beta$ gene $n$ (exp gene represents the relative expression level, $\beta$ represents the regression coefficient). To assess the prognostic role of the gene model. The ccRCC patients in the training cohort were categorized into lowrisk and high-risk groups based on the median value of the risk score, then the overall survival rate between low-risk and high-risk groups was estimated using the Kaplan Meier survival curve analysis, and differences in survival rate were compared using Log-rank test. The area under the receiver operating characteristic (ROC) curve (AUC) was calculated using the "survival ROC" R package to assess the predictive performance of prognostic model. Based on the median value of risk score from the training set, patients in the test set and entire group were also classified into low-risk and high-risk groups. Similarly, the Kaplan-Meier survival curves and ROC curves were used to test the prognostic performance of the risk model.

\section{Identification of the prognostic factors for overall survival in ccRCC}

Uni- and multivariate cox regression analyses were conducted to identify the independent prognostic factors, including age, gender, stage, grade, TNM status and risk scores of training set. A value of $p<0.05$ was regarded as statistically significant. Furthermore, we assessed the relationship between risk scores, prognosis-related hub FRGs and clinicopathological parameters of ccRCC patients in training set with Wilcoxon-signed rank test.

\section{Functional enrichment analysis of the prognostic Signature}


To illustrate the potential biological mechanisms and signaling pathways associated with the risk score, the "clusterProfiler" R package was utilized to perform $\mathrm{GO}$ and KEGG analyses based on the DEGs (Adjusted $P<0.05$ and llog2fold change $(F C) \mid \geq 1$ ) between the high-risk and low-risk groups. The cut-off criterion was Benjamin-Hochberg adjusted $p<0.05$ for all functional enrichment items.

\section{Estimation of TICs}

To investigate the relationship between risk scores and the immune microenvironment,

the content of tumor-infiltrating immune cells was calculated based on the mRNA expression matrix using the CIBERSORT algorithm. Subsequently, using the Wilcoxon-signed rank test, we performed a quantitative comparison of the distribution of TIC subtypes between high and low-risk groups in the training cohort. Likewise, we assessed the association between the risk scores with the expression levels of several immunosuppressor regulators. A P-value $<0.05$ was considered statistically significant.

\section{External validation of the prognosis-related hub FRGs}

The ONCOMINE (https://www.oncomine.org/resource/main.html) and GEPIA (http://gepia.cancerpku.cn/) database were employed to validate the expression patterns of the hub FRGs at the transcript levels. The Human Protein Atlas database (https://www.proteinatlas.org/) was used to explore the protein expression levels of hub FRGs in ccRCC. Kaplan-Meier plotter database (http://www.kmplot.com/) was utilized to assess the prognostic role of hub FRGs in ccRCC. Finally, cBioportal for Cancer Genomics (https://www.cbioportal.org/) was used to explore the genetic alterations of the prognostic genes in cCRCC.

\section{Results}

\section{DE-FRGs identification and functional enrichment analysis}

As listed in TableS1, a total of 293 ferroptosis-related genes were obtained from FerrDb database (http://www.zhounan.org/ferrdb/operations/download.html), GeneCards database (https://www.genecards.org/Search/Keyword?queryString=ferroptosis) and the previous literature. Then we obtained 71 DE-FRGs with the screening conditions mentioned above, including 34 down-regulated FRGs and 37 up-regulated FRGs (Figure 2A). Figure 2B showed the top 10 up- and down-regulated FRGs. Subsequently, we conducted the GO and KEGG analyses using the "clusterProfiler" R package to investigate the potential biological action mechanisms and signaling pathways of these DE-FRGs. As illustrated in Figure 3A, in the BP group, these DE-FRGs were mainly involved in carboxylic, organic, and unsaturated fatty acid biosynthetic process, response to hypoxia, and smooth muscle cell proliferation; in the $\mathrm{CC}$ group, these genes were significantly involved in membrane raft, membrane microdomain and apical plasma membrane; in the MF group, the DE-FRGs were enriched in oxidoreductase activity, and iron ion, coenzyme, heme and tetrapyrrole binding. Additionally, KEGG analysis revealed that several 
carcinogenic signaling pathways, such as Ferroptosis, HIF-1 signaling pathway, central carbon metabolism in cancer and MicroRNAs in cancer, were enriched among the DE-FRGs (Figure 3B)

\section{Development and validation of a prognostic ferroptosis-related gene signature}

Using univariate Cox analysis regression, totally 32 DE-FRGs significantly associated with patients' OS were identified (TableS2). Regulators of the 12 genes were remained using the LASSO regression (Figures 4A-4B), which minimizes the risk of overfitting. Subsequently, we divided all patients randomly into training set $(n=374)$ and test set $(n=156)$ according to the ratio of $7: 3$. Then, we identify 7 hub FRGs (HMGCR, MT1G, BID, EIF4A1, FOXM1, TFAP2C and CHAC1) and construct a prediction risk signature by stepwise multivariate Cox regression analysis (Figure $4 \mathrm{C}$ and Table 2). The HMGCR was considered as protective factors with HR < 1, whereas MT1G, BID, EIF4A1, FOXM1, TFAP2C and CHAC1 were risk factors with $\mathrm{HR}>1$. The risk score of each patient is calculated as the following formula: Risk score $=$ $(-0.430 * \mathrm{HMGCR})+(0.077$ * $\mathrm{MT} 1 \mathrm{G})+(0.471 * \mathrm{BID})+\left(0.318^{*}\right.$ EIF4A1 $)+(0.319$ * FOXM1) + $(0.348$ * TFAP2C $)+\left(0.364^{*} \mathrm{CHAC} 1\right)$. In addition, we evaluated the prognostic value of in ccRCC, and Kaplan-Meier survival analysis showed that overexpression of MT1G, BID, EIF4A1, FOXM1, TFAP2C, CHAC1 and low expression of HMGCR were associated with the poor OS of patients with ccRCC (Figure 4D-4J). Then, 374 cCRCC patients in the training set were divided into the low-risk group and the high-risk group according to the median value risk score. Kaplan-Meier curve analysis showed that high-risk predicted poor overall survival (Figure 5A). Figure 5B showed the distribution of the risk score, survival status of patients and heatmap plot of the seven hub FRGs between high and low risk group. ROC curve analysis showed that the area under the ROC curve for 1-year, 3-year, and 5-year OS were 0.773, 0.723, 0.731 (Figure 5C). Subsequently, patients in the testing set and the entire group were dichotomized into high-risk and lowrisk group based on the median value risk score as the cutoff in the training set. We observed that high risk score indicated a shorted overall survival rate and increased deaths both in the testing set and the entire group (Figure 6A, 6B, 7A and 7B). Additionally, in the testing set, AUC was 0.659 for 1-year OS, 0.689 for 3-year OS, and 0.783 for 5 -year OS, respectively (Figure 6C), in the whole group, AUC was 0.736 for 1-year OS, 0.712 for 3-year OS, and 0.749 for 5-year OS, respectively (Fig. 7C).

\section{Identification of independent prognostic factors}

In Univariate analysis, we found that 7-FRG based prognostic signature ( $H R=1.396, p<0.001)$, age (HR $=1.029 ; p=0.007)$, histological grade $(H R=2.603 ; p<0.001)$, clinical stage $(H R=2.091 ; p<0.001), T$ status $(H R=2.640 ; p<0.001), N$ status $(H R=4.345 ; P=0.002)$ and $M$ status $(H R=4.333 ; p<0.001)$ were associated with poorer overall survival of patients with ccRCC (Figure 8A). Furthermore, multivariate survival model after variable selection showed that age $(H R=1.066 ; p<0.001)$, $M$ status $(H R=4.548 ; p$ $=0.005)$, and the 7-FRG based prognostic signature $(H R=1.284 ; p<0.001)$ were independently associated with unfavorable OS of ccRCC patients (Figure 8B).

\section{Relationship between the hub FRGs with clinicopathological parameters}


Based on the Wilcoxon-signed rank test, we explored the association between clinicopathological parameters and the mRNA expression levels of seven hub FRGs. We found that patients with lower HMGCR expression level showed a higher histological grade (Figure 9A). As for BID, its gene expression level was correlated with clinical stage, -histological grade, and TMN classifications (Figure 9B-9F). Patients with T3-4 status had higher EIF4A1 expression than patients with T1-2 status (Figure 9G). Increased expression of FOXM1 was significantly related to a high histological grade, advanced clinical stage and high TMN status (Figure 9H-9L). Upregulation of TFAP2C expression was significantly associated with an advanced clinical stage and high T status (Figure 9M-9N). Patients with T3-4 status had higher CHAC1 expression than patients with T1-2 status (Figure 90). In addition, we observed that a high-risk score associated significantly with an advanced clinical stage, high histological grade and TMN classifications (Figure 9P-9T). However, no significant correlation was found between the MT1G expression level and clinical features of ccRCC.

\section{Functional analyses related to the 7-FRG based prognostic signature}

To explore the potential biological mechanisms and signaling pathways that were associated with the 7FRG based prognostic signature, the DEGs between the high-risk and low-risk groups were used to conduct GO and KEGG analyses. As illustrated in Figure 10A, the main biological processes (BP) involved include extracellular structure organization, epidermis development, extracellular matrix organization, negative regulation of proteolysis, hormone metabolic process, negative regulation of peptidase activity, negative regulation of endopeptidase activity, cellular hormone metabolic process, cornification and acute-phase response. The most abundant cellular component (CC) terminology was collagen-containing extracellular matrix, presynapse, endoplasmic reticulum lumen, blood microparticle, anchored component of membrane, collagen trimer, Golgi lumen, high-density lipoprotein particle, plasma lipoprotein particle and lipoprotein particle. The most abundant molecule function (MF) term was receptor ligand activity, metal ion transmembrane transporter activity, G protein-coupled receptor binding, serine hydrolase activity, serine-type endopeptidase activity, serine-type peptidase activity, cytokine activity, peptidase inhibitor activity, sodium ion transmembrane transporter activity and hormone activity. Results of the KEGG pathway analysis showed that the most abundant pathways were Neuroactive ligand-receptor interaction, Cytokine-cytokine receptor interaction, Protein digestion and absorption, IL-17 signaling pathway, Complement and coagulation cascades, Hypertrophic cardiomyopathy, Arachidonic acid metabolism, Mineral absorption, Linoleic acid metabolism and Phototransduction (Figure 10B).

\section{Association of the 7-FRG based prognostic signature with tumor-infiltrating immune cells and immune checkpoints}

To uncover the relationship between the FRG-based prognostic model and tumor immune microenvironment (TME), we explored the difference of tumor-infiltrating immune cells between two groups. The results showed the abundance of Dendritic cells resting, Macrophages M2, Mast cells resting and Monocytes were significantly enriched in the low-risk group (Figure 11A-11D). However, Macrophages 
M0, Plasma cells, T cells follicular helper and T cells regulatory (Tregs) enriched more in the high-risk group (Figure 11E-11H). Tumor cells could escape from immune response by activating the immune checkpoint pathway. Thus, we estimated the association between the expression level of immune checkpoints and the FRG-based prognostic model, and results showed that PDCD1, CTLA4, LAG3, CD276 and TIGIT were highly expressed in patients with high-risk score (Figure 12A-12E). These results collectively suggest that the changes in the proportion of immune infiltrating cells and the expression of crucial immune checkpoints might be associate with the risk stratification of ccRCC.

\section{External verification of the hub FRGs}

Using the ONCOMINE database, we found that HMGCR, MT1G, TFAP2C and CHAC1 were significantly down-regulated in ccRCC samples by comparison with normal renal samples, whereas BID and EIF4A1 were highly expressed in ccRCC specimens compared to noncancerous specimens (Figure 13A and Table 3). However, no difference of FOXM1 relative expression was found between ccRCC tissues and healthy control tissues. We also evaluated the expression levels of the hub FRGs in ccRCC samples and normal samples with GEPIA. Results showed that MT1G and CHAC1 were significantly lower in CcRCC samples than normal renal samples, BID and FOXM1 were significantly higher in cCRCC specimens than noncancerous specimens, however, there was no significant difference in HMGCR, EIF4A1 and TFAP2C expression between ccRCC and normal renal tissues (Figure 13B-13H). In addition, the representative protein expression of HMGCR, BID, EIF4A1, FOXM1, TFAP2C and CHAC1 was investigate in the Human Protein Profiles and shown in Figure 13I-13N. However, MT1G was not found on the website. The value of the hub FRGs in the overall survival of ccRCC patients was also assessed using the Kaplan-Meier plotter database, and results showed that overexpression of MT1G, BID, EIF4A1, FOXM1, TFAP2C, CHAC1 and low expression of HMGCR were related to the poor OS of patients with CCRCC (130-13U), which are consistent with our above results. Furthermore, we analyzed the genetic alterations of the hub FRGs in ccRCC using the cBioportal database and results were exhibited in Figure 13V.

\section{Discussion}

Unlike autophagy and apoptosis, ferroptosis, a novel form of regulated cell death, has unique characteristics in morphology, biochemistry, and genetics ${ }^{[9,10]}$. Mechanistic studies have suggested that system XC $\mathrm{XC}^{-}$inhibition, GSH depletion or GPX4 inhibition, and iron-dependent ROS accumulation would induce cellular ferroptosis. Accumulating evidence have indicated that ferroptosis played a vital role in tumor biology ${ }^{[15]}$. It has recently been shown that promotion of ferroptosis could be exploited to treat cancer, and ferroptosis inducers exhibited great potential to improve therapeutic performance ${ }^{[16,19]}$. For instance, Markowitsch et al have demonstrated that artesunate could inhibit growth of sunitinib-resistant renal cell carcinoma cells through cell cycle arrest and induction of ferroptosis ${ }^{[20]}$. Sulfasalazine, a system xc- inhibitor, could inhibit cysteine uptake and lead to ferroptosis of glioma cells ${ }^{[21]}$. Cisplatin was recognized as a ferroptosis-inducer, which could reduce GSH depletion and GPXs inactivation, resulting in ferroptosis in $\mathrm{A} 549$ and HCT116 cells ${ }^{[22]}$. Therefore, ferroptosis -related biomarkers exhibit great potential 
for risk stratification and for exerting prognostic value. In previous studies, ferroptosis-related signatures have been identified as independent prognostic factors in several malignancies ${ }^{[23-26]}$, however, the potential value of ferroptosis-related signature for predicting prognosis in ccRCC is lacking.

Herein, we established a novel predictive model based on seven FRGs, which performed well in stratifying the risk groups of cCRCC in both TCGA training cohort, testing cohort and the entire group. Uni- and multivariate Cox analysis suggested that the 7-FRG based prognostic signature was independent of other clinicopathological variables. The prognostic model in our study was composed of a protective gene (HMGCR) and six risk-related genes FRGs (MT1G, BID, EIF4A1, FOXM1, TFAP2C and CHAC1). HMGCR was an enzyme involved in cholesterol synthesis, Kenichi Shimada et al have reported that HMGCR inhibition could enhanced FIN56 lethality (a new specific ferroptosis-inducer) and sensitivity to FIN56induced ferroptosis ${ }^{[27]}$. In HCC cells, MT1G have been identified as an accomplice of sorafenib resistance, MT-1G knockdown by RNA interference significantly increased glutathione depletion and lipid peroxidation, which result in sorafenib-induced ferroptosis ${ }^{[28]}$. BID was a member of the BCL-2 family of cell death regulators, knockdown of BID inhibited pharmacologically induced ferroptosis ${ }^{[29]}$. Circ_0008035 promoted cell proliferation and inhibited apoptosis and ferroptosis in gastric cancer through regulating the miR-599/EIF4A1 axis, increased EIF4A1 expression levels partially reversed the effects of circ_0008035 deficiency on iron accumulation, lipid peroxidation and mitochondrial membrane potential in GC cells ${ }^{[30]}$. FOXM1 was a crucial transcriptional regulator of Forkhead box (FOX) superfamily, inhibition of FOXM1 increased erastin-induced ferroptosis in in melanoma cells ${ }^{[31]}$. TFAP2C was a member of the retinoic acid-inducible AP-2 factor, which played an important role in cell differentiation, tissue development and tumor biology, it could positively regulate the expression of GPX4, ultimately inhibiting GPX4-dependent ferroptotic death ${ }^{[32]}$. CHAC1, a member of the gamma-

glutamylcyclotransferase family of proteins, play an important role in regulation of glutathione levels and oxidative balance in the cell[33], and previous studies have shown that overexpression of CHAC1 promoted ferroptosis in Burkitt's Lymphoma ${ }^{[34]}$. However, whether HMGCR, MT1G, BID, EIF4A1, FOXM1, TFAP2C and CHAC1 played roles in the occurrence and development of ccRCC by affecting the ferroptosis remains to be addressed, relevant studies are very less.

Based on the DEGs between different risk groups, GO and KEGG analyses were conducted to uncover the potential biological mechanisms and pathways associated this prognostic signature, and we found that several carcinogenic pathways were enriched, which included some immune-related signaling pathways, such as the IL-17 signaling pathway and cytokine-cytokine receptor interaction. They were both crucial immune signaling pathways, with the former could promote M2 polarization of tumor-associated macrophages (TAMs) via the NF-kB signaling pathway, providing important support for tumor progression and metastasi ${ }^{[35]}$, and the latter could regulate cytokine interactions and thus promoting cancer development and progression ${ }^{[36]}$. Considerable evidence showed that the immune microenvironment played a crucial role in the carcinogenesis and progression of malignancies ${ }^{[37]}$. In the study, we assessed the the profiles of immune cell composition in high- and low-risk groups, and results showed that ccRCC patients with high-risk scores identified by the ferroptosis and iron metabolism 
signatures had higher proportions of Macrophages M0, Plasma cells, T cells follicular helper and T cells regulatory (Tregs), suggesting a regulatory influence on tumor immune microenvironment, and also indicating that stronger immunosuppressive effect in ccRCC patients with high-risk scores may be an important cause for their poor prognosis. In addition, we confirmed that immune checkpoints, such as PDCD1, CTLA4, LAG3, CD276 and TIGIT, were highly expressed in the high-risk group by comparison with low-risk group. These differences avoid immune surveillance and further promote the growth and progression of $\mathrm{ccRCC}$, also suggested that patients with high-risk scores might benefit more from immune checkpoint inhibitors.

In conclusion, we confirmed that risk signature based on seven ferroptosis-related genes might be effective in risk classification and able to serve as an independent prognostic factor for the overall survival of patients with ccRCC. However, further in vitro and in vivo experiments must be carried out to investigate the potential action mechanisms behind ferroptosis-related genes and the survival outcome of ccRCC.

\section{Declarations}

Ethical approval and consent to participate: Not applicable.

Consent for publication: Not applicable.

Availability of data and materials: The results were often aggregated from multiple databases and analyzed online without the need to download the data. The data of the $530 \mathrm{ccRCC}$ patients from TCGA database were downloaded from https://portal.gdc.cancer.gov/.

Competing interests: The authors declare that they have no competing interests, and all authors confirm its accuracy.

Funding: Not applicable.

Authors' contributions: (I) Conception and design: Yen-ting Lin; (II) Administrative support: Can-Xuan Li, Yen-ting Lin, Jie Chen; (III) Provision of study materials or patients: Can-Xuan Li; (IV) Collection and assembly of data: Can-Xuan Li, Yen-ting Lin, Jie Chen; (V) Data analysis and interpretation: Can-Xuan Li, Yen-ting Lin; (VI) Manuscript writing: All authors; (VII) Final approval of manuscript: All authors.

Acknowledgments: Not applicable.

Authors' information: Yen-ting Lin ${ }^{1}$, Can-Xuan Li ${ }^{1}$, Jie Chen ${ }^{1}$

${ }^{1}$ Department of Urology, The First Affiliated Hospital of Jinan University, Guang Zhou, Guang Dong, China.

First author: Yen-ting Lin: E-mail: yenting_lin@163.com 
Corresponding author: Can-Xuan Li: E-mail: china20170713@163.com

\section{References}

1. SIEGEL R L, MILLER K D. Cancer statistics, 2020 [J]. 2020, 70(1): 7-30.

2. HSIEH J J, PURDUE M P, SIGNORETTI S, et al. Renal cell carcinoma [J]. Nat Rev Dis Primers, 2017, 3(17009-.

3. VERA-BADILLO F E, TEMPLETON A J, DURAN I, et al. Systemic therapy for non-clear cell renal cell carcinomas: a systematic review and meta-analysis [J]. European urology, 2015, 67(4): 740-9.

4. CAPITANIO U, MONTORSI F. Renal cancer [J]. Lancet (London, England), 2016, 387(10021): 894-906.

5. ATKINS M B, TANNIR N M. Current and emerging therapies for first-line treatment of metastatic clear cell renal cell carcinoma [J]. Cancer treatment reviews, 2018, 70(127-37.

6. BARATA P C, RINI B I. Treatment of renal cell carcinoma: Current status and future directions [J]. CA Cancer J Clin, 2017, 67(6): 507-24.

7. POSADAS E M, LIMVORASAK S, FIGLIN R A. Targeted therapies for renal cell carcinoma [J]. Nature reviews Nephrology, 2017, 13(8): 496-511.

8. RINI B I, BATTLE D, FIGLIN R A, et al. The society for immunotherapy of cancer consensus statement on immunotherapy for the treatment of advanced renal cell carcinoma (RCC) [J]. 2019, 7(1): 354.

9. DIXON S J, LEMBERG K M, LAMPRECHT M R, et al. Ferroptosis: an iron-dependent form of nonapoptotic cell death [J]. Cell, 2012, 149(5): 1060-72.

10. GREEN D R, VICTOR B. The pantheon of the fallen: why are there so many forms of cell death? [J]. Trends in cell biology, 2012, 22(11): 555-6.

11. SHEN Z, SONG J, YUNG B C, et al. Emerging Strategies of Cancer Therapy Based on Ferroptosis [J]. 2018, 30(12): e1704007.

12. LANG X, GREEN M D. Radiotherapy and Immunotherapy Promote Tumoral Lipid Oxidation and Ferroptosis via Synergistic Repression of SLC7A11 [J]. 2019, 9(12): 1673-85.

13. GASCHLER M M, ANDIA A A, LIU H, et al. FINO(2) initiates ferroptosis through GPX4 inactivation and iron oxidation [J]. 2018, 14(5): 507-15.

14. BR TSCH S H, WANG C C, LI L, et al. Expression of inactive glutathione peroxidase 4 leads to embryonic lethality, and inactivation of the Alox15 gene does not rescue such knock-in mice [J]. Antioxidants \& redox signaling, 2015, 22(4): 281-93.

15. MOU Y, WANG J, WU J, et al. Ferroptosis, a new form of cell death: opportunities and challenges in cancer [J]. Journal of hematology \& oncology, 2019, 12(1): 34.

16. LIANG C, ZHANG X. Recent Progress in Ferroptosis Inducers for Cancer Therapy [J]. 2019, 31(51): e1904197.

17. NIE J, LIN B, ZHOU M, et al. Role of ferroptosis in hepatocellular carcinoma [J]. 2018, 144(12): 232937. 
18. MA S, HENSON E S, CHEN Y, et al. Ferroptosis is induced following siramesine and lapatinib treatment of breast cancer cells [J]. Cell Death Dis, 2016, 7(7): e2307.

19. WANG Y, WEI Z, PAN K, et al. The function and mechanism of ferroptosis in cancer [J]. 2020, 25(1112): 786-98.

20. MARKOWITSCH S D, SCHUPP P, LAUCKNER J, et al. Artesunate Inhibits Growth of Sunitinib-Resistant Renal Cell Carcinoma Cells through Cell Cycle Arrest and Induction of Ferroptosis [J]. Cancers, 2020, 12(11):

21. TOYOKUNI S, ITO F, YAMASHITA K, et al. Iron and thiol redox signaling in cancer: An exquisite balance to escape ferroptosis [J]. Free radical biology \& medicine, 2017, 108(610-26.

22. GUO J, XU B, HAN Q, et al. Ferroptosis: A Novel Anti-tumor Action for Cisplatin [J]. Cancer research and treatment, 2018, 50(2): 445-60.

23. LIU H J, HU H M, LI G Z, et al. Ferroptosis-Related Gene Signature Predicts Glioma Cell Death and Glioma Patient Progression [J]. Frontiers in cell and developmental biology, 2020, 8(538.

24. TANG B, ZHU J, LI J, et al. The ferroptosis and iron-metabolism signature robustly predicts clinical diagnosis, prognosis and immune microenvironment for hepatocellular carcinoma [J]. 2020, 18(1): 174.

25. TANG R, HUA J, XU J, et al. The role of ferroptosis regulators in the prognosis, immune activity and gemcitabine resistance of pancreatic cancer [J]. Annals of translational medicine, 2020, 8(21): 1347.

26. GAO X, TANG M. A ferroptosis-related gene signature predicts overall survival in patients with lung adenocarcinoma [J]. 2021,

27. SHIMADA K, SKOUTA R, KAPLAN A, et al. Global survey of cell death mechanisms reveals metabolic regulation of ferroptosis [J]. 2016, 12(7): 497-503.

28. SUN X, NIU X, CHEN R, et al. Metallothionein-1G facilitates sorafenib resistance through inhibition of ferroptosis [J]. Hepatology (Baltimore, Md), 2016, 64(2): 488-500.

29. NEITEMEIER S, JELINEK A, LAINO V, et al. BID links ferroptosis to mitochondrial cell death pathways [J]. Redox biology, 2017, 12(558-70.

30. LI C, TIAN Y, LIANG Y, et al. Circ_0008035 contributes to cell proliferation and inhibits apoptosis and ferroptosis in gastric cancer via miR-599/EIF4A1 axis [J]. Cancer cell international, 2020, 20(84.

31. YANG Y, LUO M, ZHANG K. Nedd4 ubiquitylates VDAC2/3 to suppress erastin-induced ferroptosis in melanoma [J]. 2020, 11(1): 433.

32. ALIM I, CAULFIELD J T, CHEN Y, et al. Selenium Drives a Transcriptional Adaptive Program to Block Ferroptosis and Treat Stroke [J]. Cell, 2019, 177(5): 1262-79.e25.

33. BACHHAWAT A K, KAUR A. Glutathione Degradation [J]. Antioxidants \& redox signaling, 2017, 27(15): 1200-16.

34. WANG N, ZENG G Z, YIN J L, et al. Artesunate activates the ATF4-CHOP-CHAC1 pathway and affects ferroptosis in Burkitt's Lymphoma [J]. Biochemical and biophysical research communications, 2019, 519(3): 533-9. 
35. LI X, BECHARA R, ZHAO J, et al. IL-17 receptor-based signaling and implications for disease [J]. 2019, 20(12): 1594-602.

36. SPANGLER J B, MORAGA I, MENDOZA J L, et al. Insights into cytokine-receptor interactions from cytokine engineering [J]. Annual review of immunology, 2015, 33(139-67.

37. GAJEWSKI T F, SCHREIBER H, FU Y X. Innate and adaptive immune cells in the tumor microenvironment [J]. Nature immunology, 2013, 14(10): 1014-22.

\section{Tables}

Table 1. Characteristics of clear cell renal cell carcinoma patients in the TCGA database. 


\begin{tabular}{|c|c|c|c|}
\hline Clinical parameters & Variable & Total $(n=530)$ & Percentages (\%) \\
\hline \multirow[t]{2}{*}{ age } & $\leq 60$ & 264 & 49.81 \\
\hline & $\llbracket 60$ & 266 & 50.19 \\
\hline \multirow[t]{2}{*}{ gender } & female & 186 & 35.09 \\
\hline & male & 344 & 64.91 \\
\hline \multirow[t]{6}{*}{ Histological grade } & $\mathrm{G} 1$ & 14 & 2.64 \\
\hline & $\mathrm{G} 2$ & 227 & 42.83 \\
\hline & G3 & 206 & 38.87 \\
\hline & $\mathrm{G} 4$ & 75 & 14.15 \\
\hline & $\mathrm{GX}$ & 5 & 0.94 \\
\hline & unknow & 3 & 0.57 \\
\hline \multirow[t]{5}{*}{ clinical stage } & stage I & 265 & 50 \\
\hline & stage II & 57 & 10.74 \\
\hline & stage III & 123 & 23.21 \\
\hline & stage IV & 82 & 15.48 \\
\hline & unknow & 3 & 0.57 \\
\hline \multirow[t]{4}{*}{ T classification } & $\mathrm{T} 1$ & 271 & 51.13 \\
\hline & $\mathrm{T} 2$ & 69 & 13.02 \\
\hline & T3 & 179 & 33.77 \\
\hline & $\mathrm{T} 4$ & 11 & 2.08 \\
\hline \multirow[t]{4}{*}{ Distant metastasis } & MO & 420 & 79.25 \\
\hline & M1 & 78 & 14.72 \\
\hline & $M X$ & 30 & 5.66 \\
\hline & unknow & 2 & 0.37 \\
\hline \multirow[t]{3}{*}{ Lymph nodes } & NO & 239 & 45.09 \\
\hline & N1 & 16 & 3.02 \\
\hline & $\mathrm{NX}$ & 275 & 51.89 \\
\hline
\end{tabular}

TCGA, The Cancer Genome Atlas. 
Table 2. Multivariate Cox regression analysis to identify prognosis related hub FRGs

\begin{tabular}{|lllllll|}
\hline Id & coef & HR & HR.95L & HR.95H & pvalue & Expression \\
\hline HMGCR & -0.430 & 0.650 & 0.414 & 1.022 & 0.062 & down \\
\hline MT1G & 0.077 & 1.080 & 0.983 & 1.187 & 0.111 & down \\
\hline BID & 0.471 & 1.602 & 0.939 & 2.731 & 0.084 & up \\
\hline EIF4A1 & 0.318 & 1.375 & 1.076 & 1.758 & 0.011 & up \\
\hline FOXM1 & 0.319 & 1.375 & 1.083 & 1.746 & 0.009 & up \\
\hline TFAP2C & 0.348 & 1.416 & 0.999 & 2.006 & 0.051 & down \\
\hline CHAC1 & 0.364 & 1.439 & 1.141 & 1.816 & 0.002 & down \\
\hline
\end{tabular}

FRGs, ferroptosis-related genes

Table 3. The significant changes of hub FRGs expression in transcription level between different types of ccRCC and normal renal tissues (Oncomine database). 


\begin{tabular}{|c|c|c|c|c|}
\hline Gene & Types of ccRCC vs. normal samples & $\begin{array}{l}\text { Fold } \\
\text { change }\end{array}$ & $P$ value & t-test \\
\hline HMGCR & $\begin{array}{l}\text { Non-Hereditary Clear Cell Renal Cell Carcinoma vs. } \\
\text { Normal }\end{array}$ & -2.103 & $\begin{array}{l}2.13 \mathrm{E}- \\
07\end{array}$ & -6.672 \\
\hline \multirow[t]{6}{*}{ MT1G } & Clear Cell Renal Cell Carcinoma vs. Normal & -8.244 & $\begin{array}{l}1.33 \mathrm{E}- \\
11\end{array}$ & -13.545 \\
\hline & Clear Cell Renal Cell Carcinoma vs. Normal & -6.018 & $\begin{array}{l}1.61 \mathrm{E}- \\
05\end{array}$ & -6.857 \\
\hline & Clear Cell Renal Cell Carcinoma vs. Normal & -2.029 & $\begin{array}{l}7.22 \mathrm{E}- \\
14\end{array}$ & -12.127 \\
\hline & $\begin{array}{l}\text { Non-Hereditary Clear Cell Renal Cell Carcinoma vs. } \\
\text { Normal }\end{array}$ & -13.728 & $\begin{array}{l}1.49 \mathrm{E}- \\
08\end{array}$ & -9.055 \\
\hline & $\begin{array}{l}\text { Hereditary Clear Cell Renal Cell Carcinoma vs. } \\
\text { Normal }\end{array}$ & -16.308 & $\begin{array}{l}2.81 \mathrm{E}- \\
08\end{array}$ & -10.545 \\
\hline & Clear Cell Renal Cell Carcinoma vs. Normal & -9.757 & $\begin{array}{l}7.40 \mathrm{E}- \\
07\end{array}$ & -10.348 \\
\hline \multirow[t]{3}{*}{ BID } & $\begin{array}{l}\text { Non-Hereditary Clear Cell Renal Cell Carcinoma vs. } \\
\text { Normal }\end{array}$ & 3.084 & $\begin{array}{l}4.84 \mathrm{E}- \\
09\end{array}$ & 9.461 \\
\hline & $\begin{array}{l}\text { Hereditary Clear Cell Renal Cell Carcinoma vs. } \\
\text { Normal }\end{array}$ & 2.900 & $\begin{array}{l}3.20 \mathrm{E}- \\
08\end{array}$ & 9.623 \\
\hline & Clear Cell Renal Cell Carcinoma vs. Normal & 2.127 & $\begin{array}{l}1.00 \mathrm{E}- \\
03\end{array}$ & 5.642 \\
\hline \multirow[t]{2}{*}{ EIF4A1 } & $\begin{array}{l}\text { Non-Hereditary Clear Cell Renal Cell Carcinoma vs. } \\
\text { Normal }\end{array}$ & 2.172 & $\begin{array}{l}1.16 \mathrm{E}- \\
08\end{array}$ & 9.959 \\
\hline & Clear Cell Renal Cell Carcinoma vs. Normal & 2.416 & $\begin{array}{l}6.33 \mathrm{E}- \\
10\end{array}$ & 7.945 \\
\hline FOXM1 & NA & NA & NA & NA \\
\hline TFAP2C & Clear Cell Renal Cell Carcinoma vs. Normal & -4.357 & $\begin{array}{l}4.36 \mathrm{E}- \\
05\end{array}$ & -4.722 \\
\hline RPL18A & Clear Cell Renal Cell Carcinoma vs. Normal & -2.566 & $\begin{array}{l}5.00 \mathrm{E}- \\
03\end{array}$ & -3.105 \\
\hline
\end{tabular}

FRGs, ferroptosis-related genes; ccRCC, clear cell renal cell carcinoma.

\section{Figures}




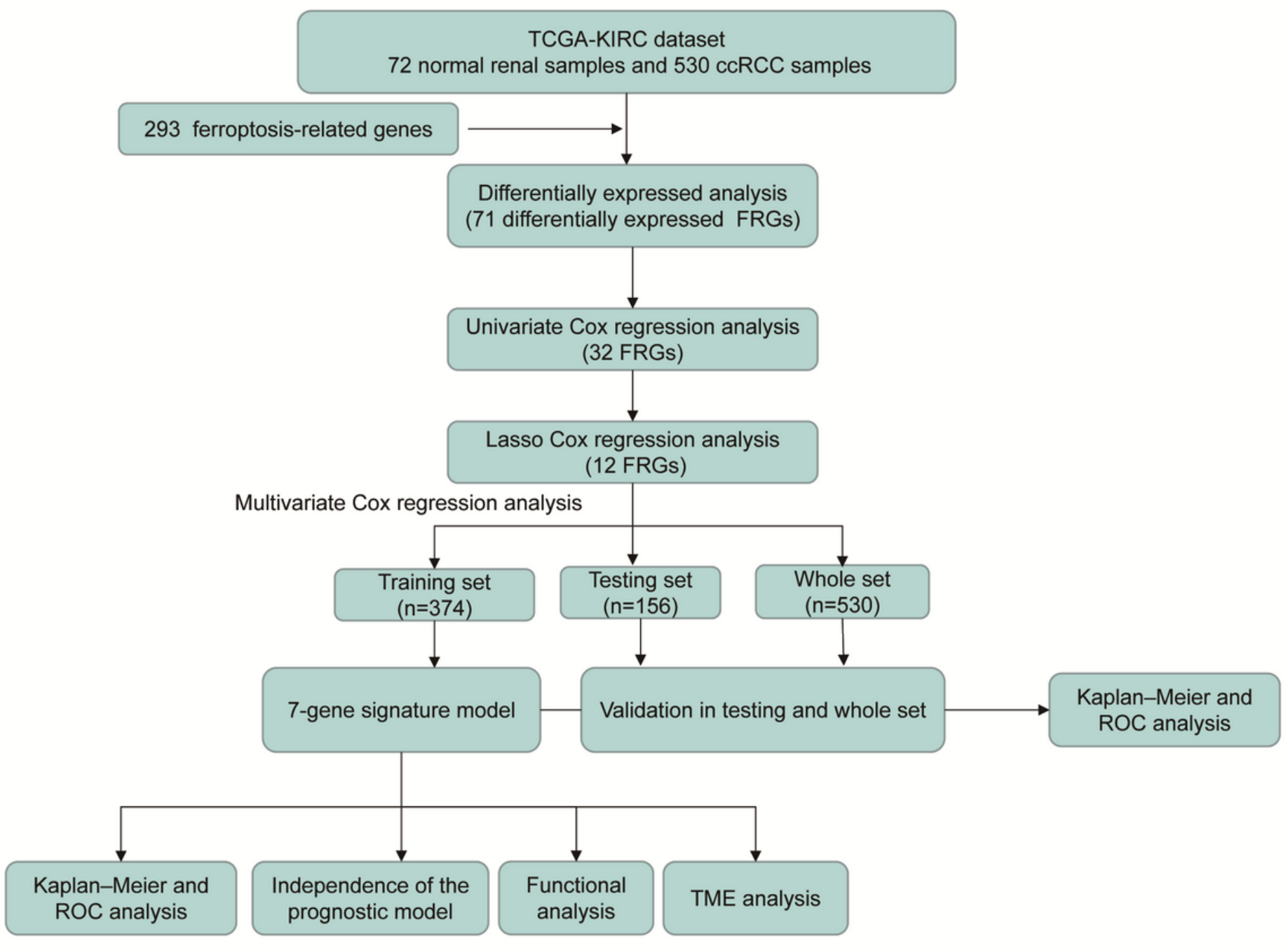

\section{Figure 1}

Flow chart of the study. 
A

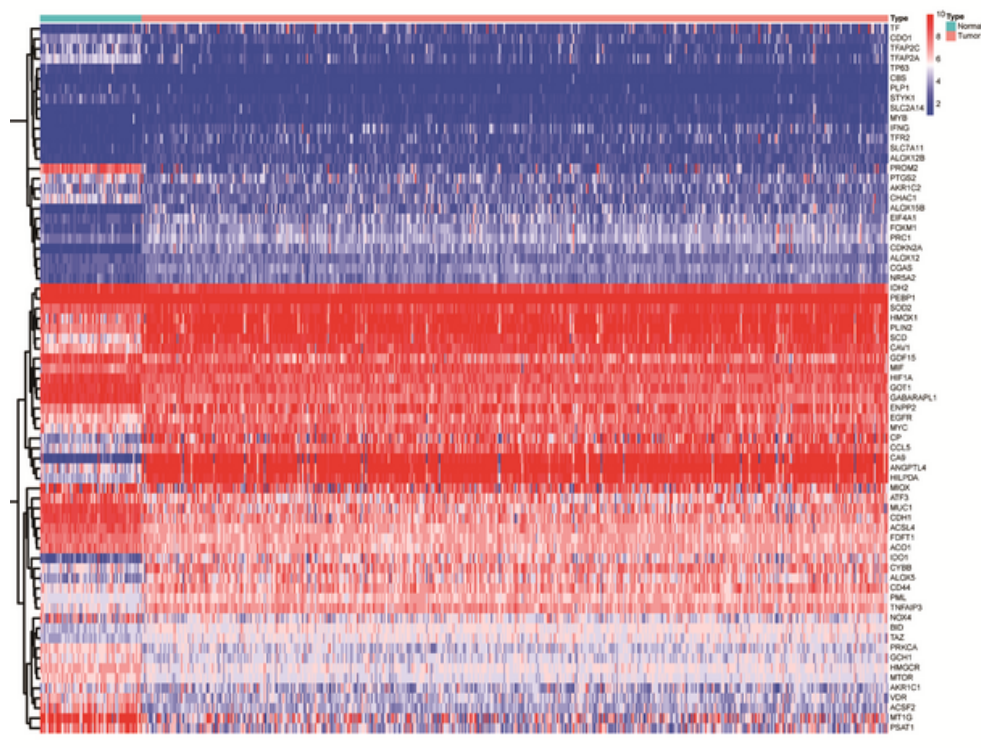

B

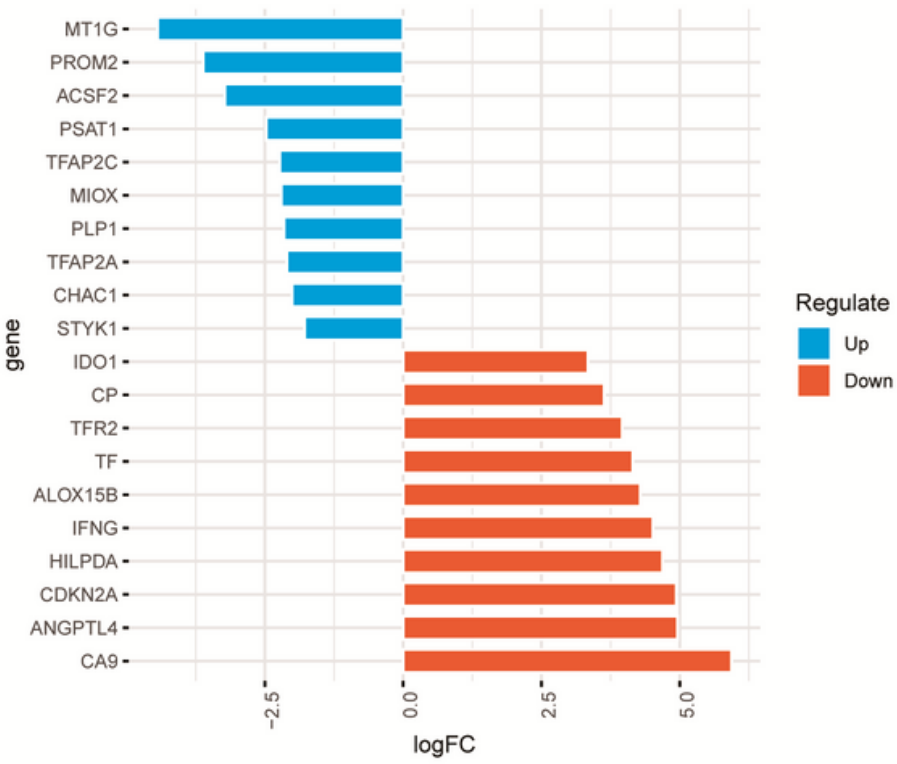

Figure 2

Identification of differentially expressed ferroptosis-related genes (DE-FRGs) between normal tissues and ccRCC tissues. (A) The heat map of DE-FRGs; (B) The top 10 up-regulated and down-regulated DE-FRGs. ccRCC, clear cell renal cell carcinoma. 
A

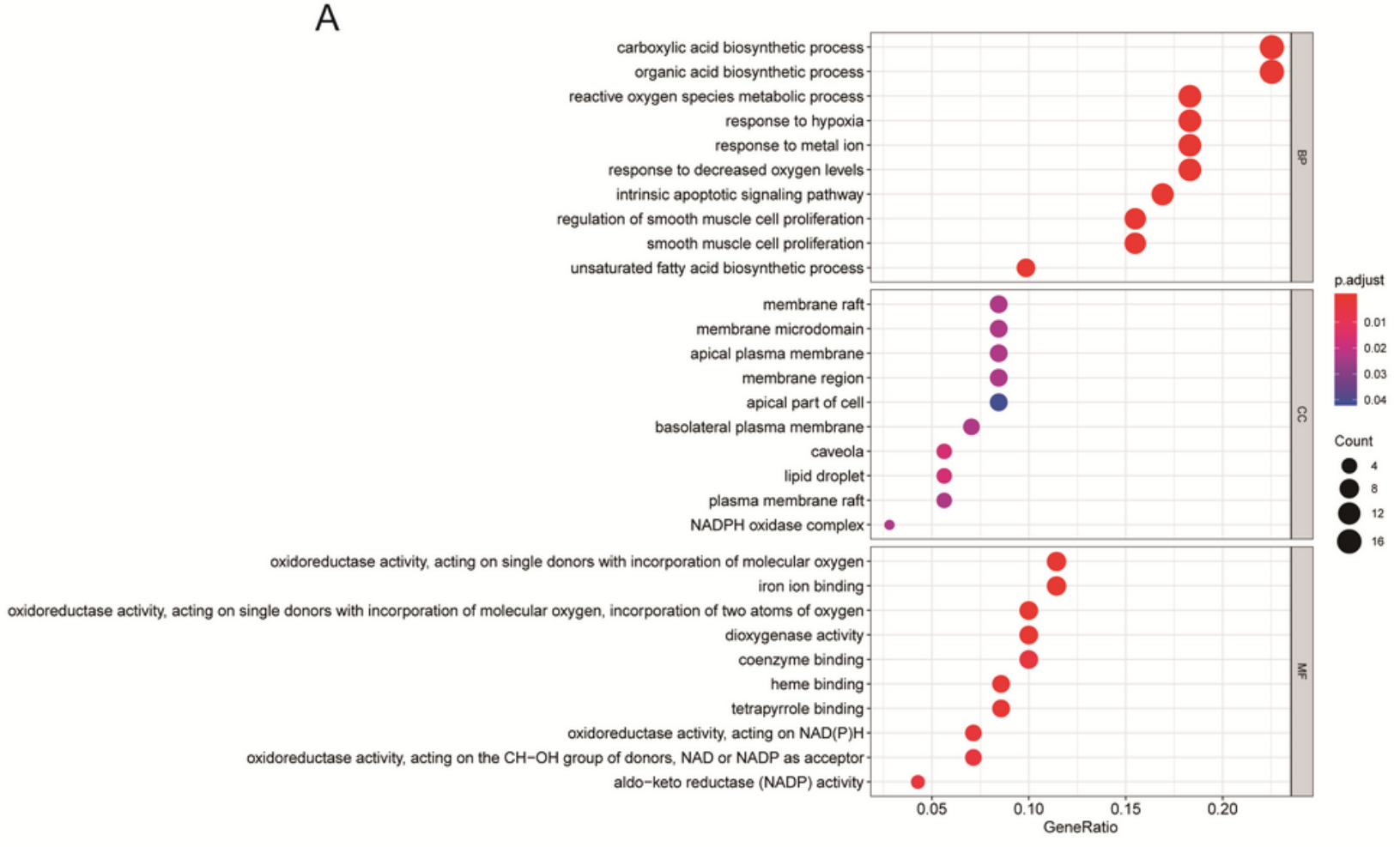

B

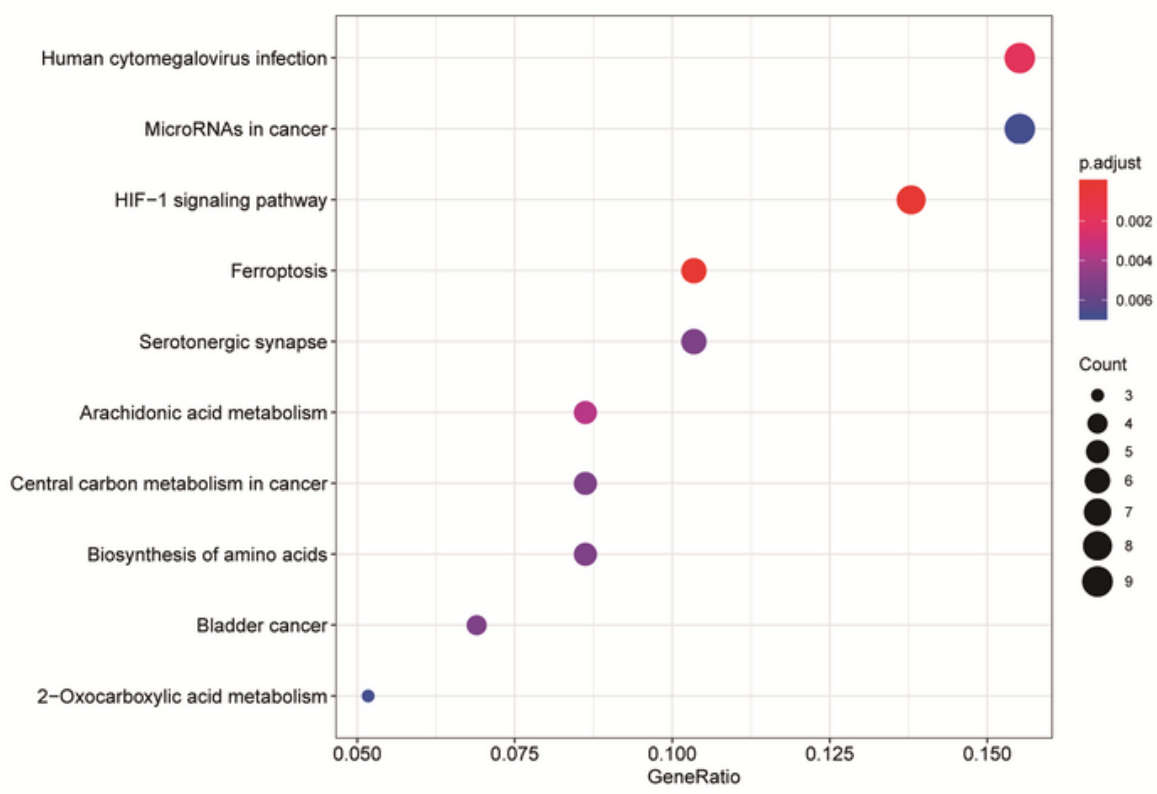

\section{Figure 3}

Representative results of GO and KEGG. (A) Significantly enriched GO terms of DE-FRGs; (B) KEGG signaling pathway analysis of DE-FRGs. GO, Gene Ontology; BP, biological process; CC, cellular component; MF, molecular function; KEGG, Kyoto Encyclopedia of Genes and Genomes pathways; DEFRGs, differentially expressed ferroptosis-related genes. 
A

B
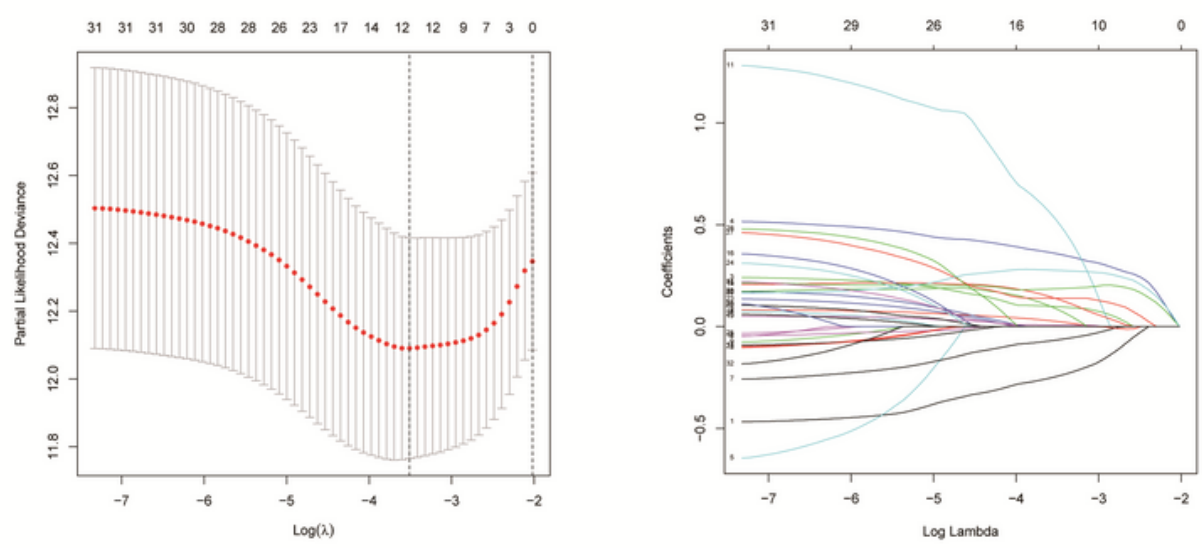

C

Hazard ratio

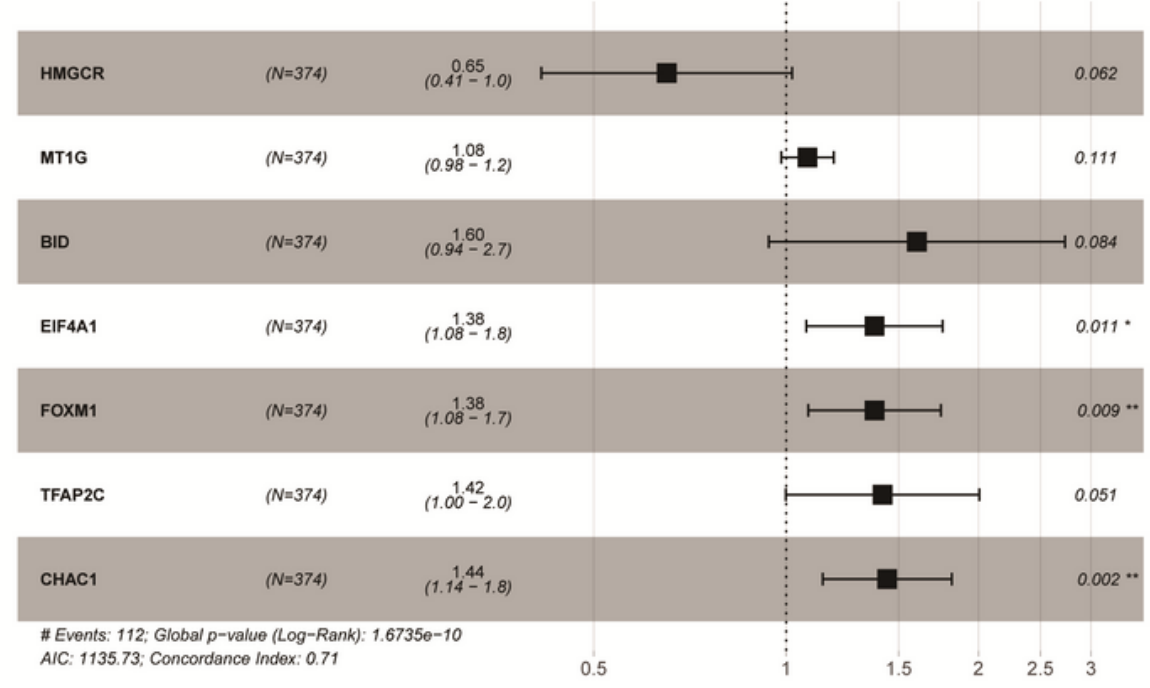
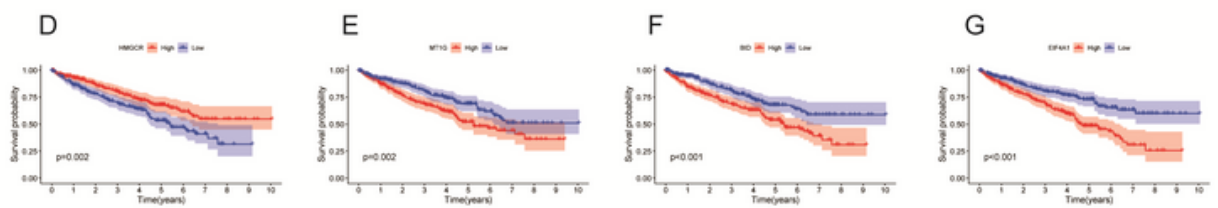

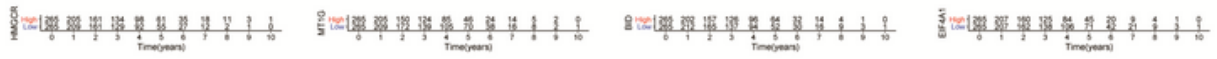
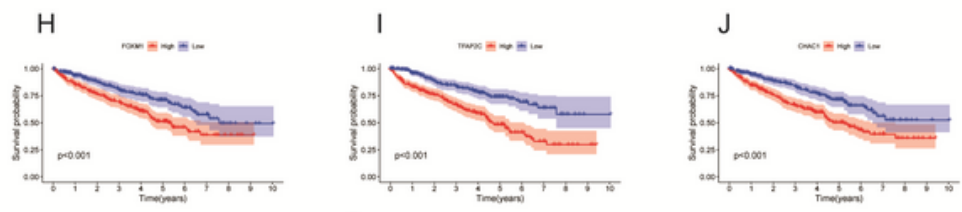

51:

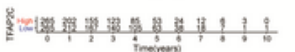

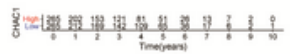

\section{Figure 4}

Identification of prognosis related hub FRGs. (A, B) LASSO regression identified 12 FRGs; (C) Multivariate Cox regression analysis to identify prognosis related hub FRGs; (D-J) Kaplan-Meier survival curves of seven hub FRGs. FRGs, ferroptosis-related genes; LASSO, Least absolute shrinkage and selection operator. 
A

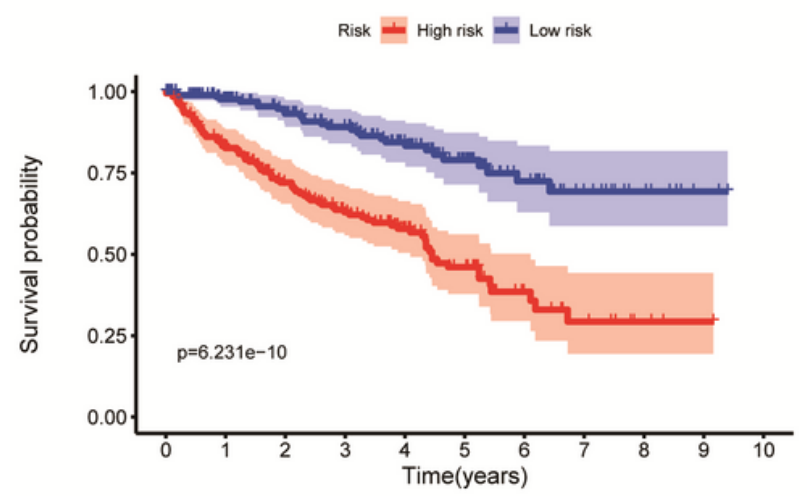

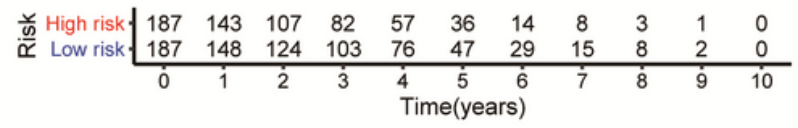

C

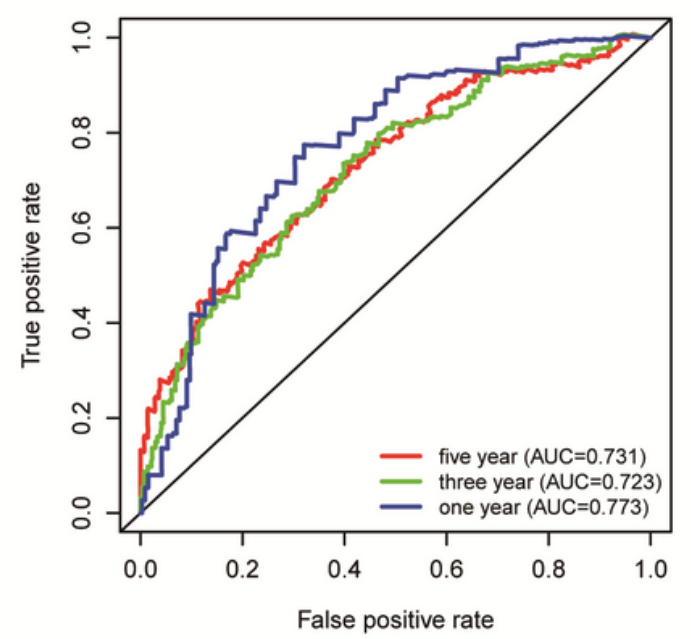

B
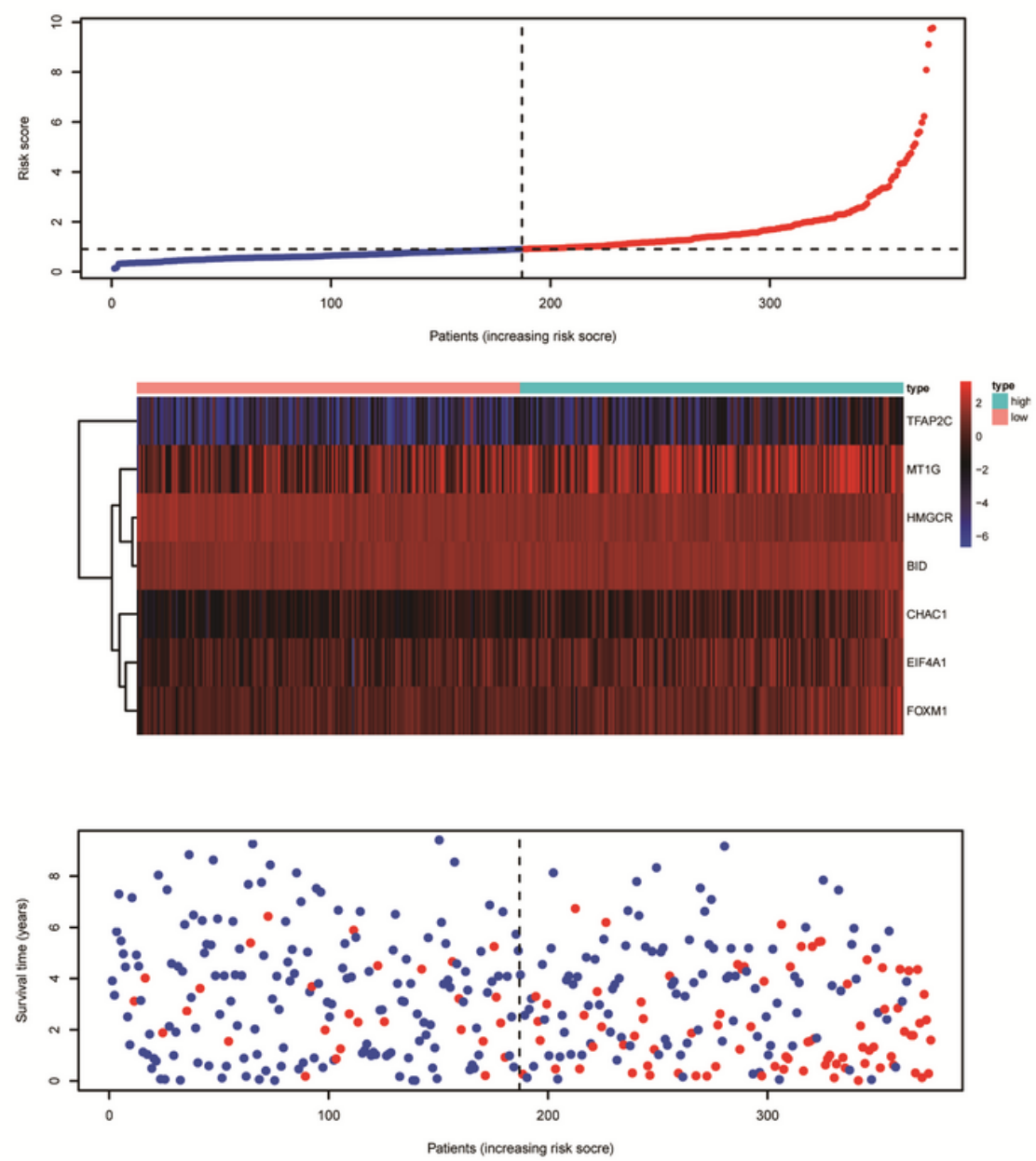

\section{Figure 5}

Development of the prognostic signature based on seven ferroptosis-related genes (FRGs) in the TCGA training set. (A) Survival analysis between signature-defined risk groups; (B) Relationship among the risk score (upper), the expression profiles of the seven prognostic genes (middle), and survival status of patients in different groups (bottom) in the training dataset. (C) Time-dependent ROC curve of the 7-FRG prognostic signature. ROC, receiver operating characteristic curve; TCGA, The Cancer Genome Atlas. 
A

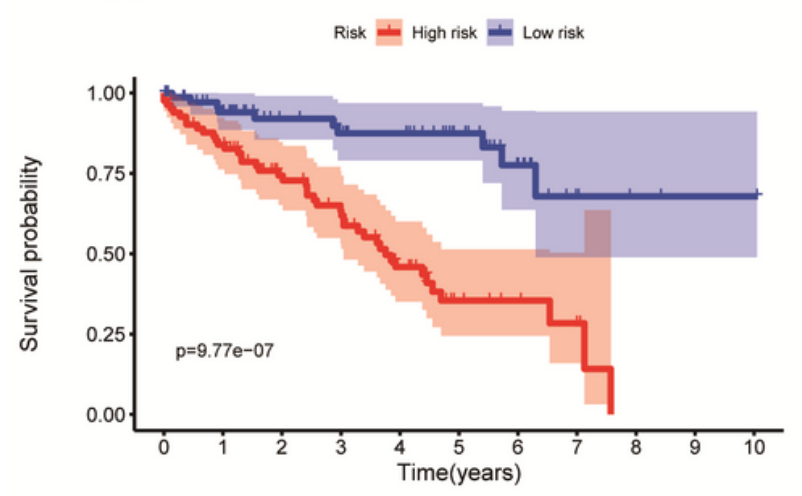

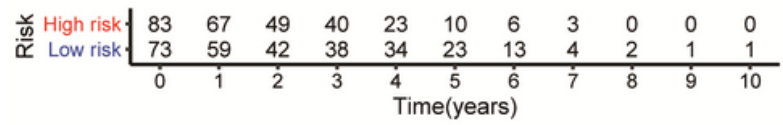

C

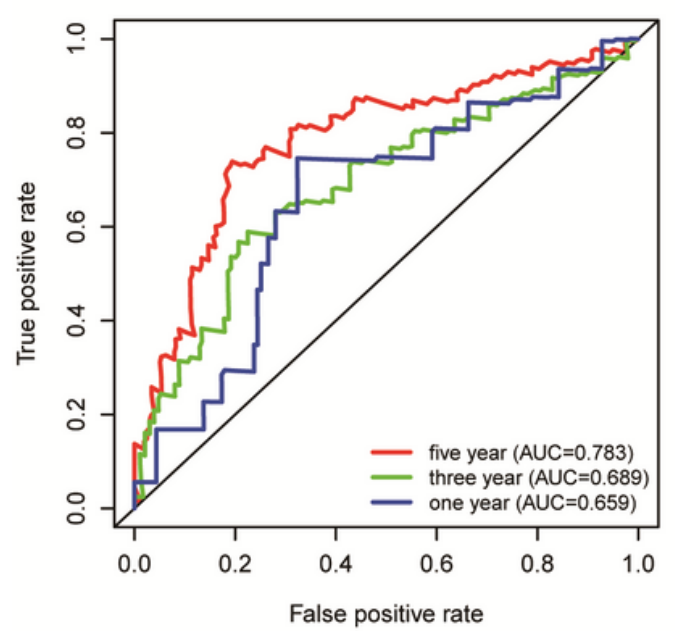

B
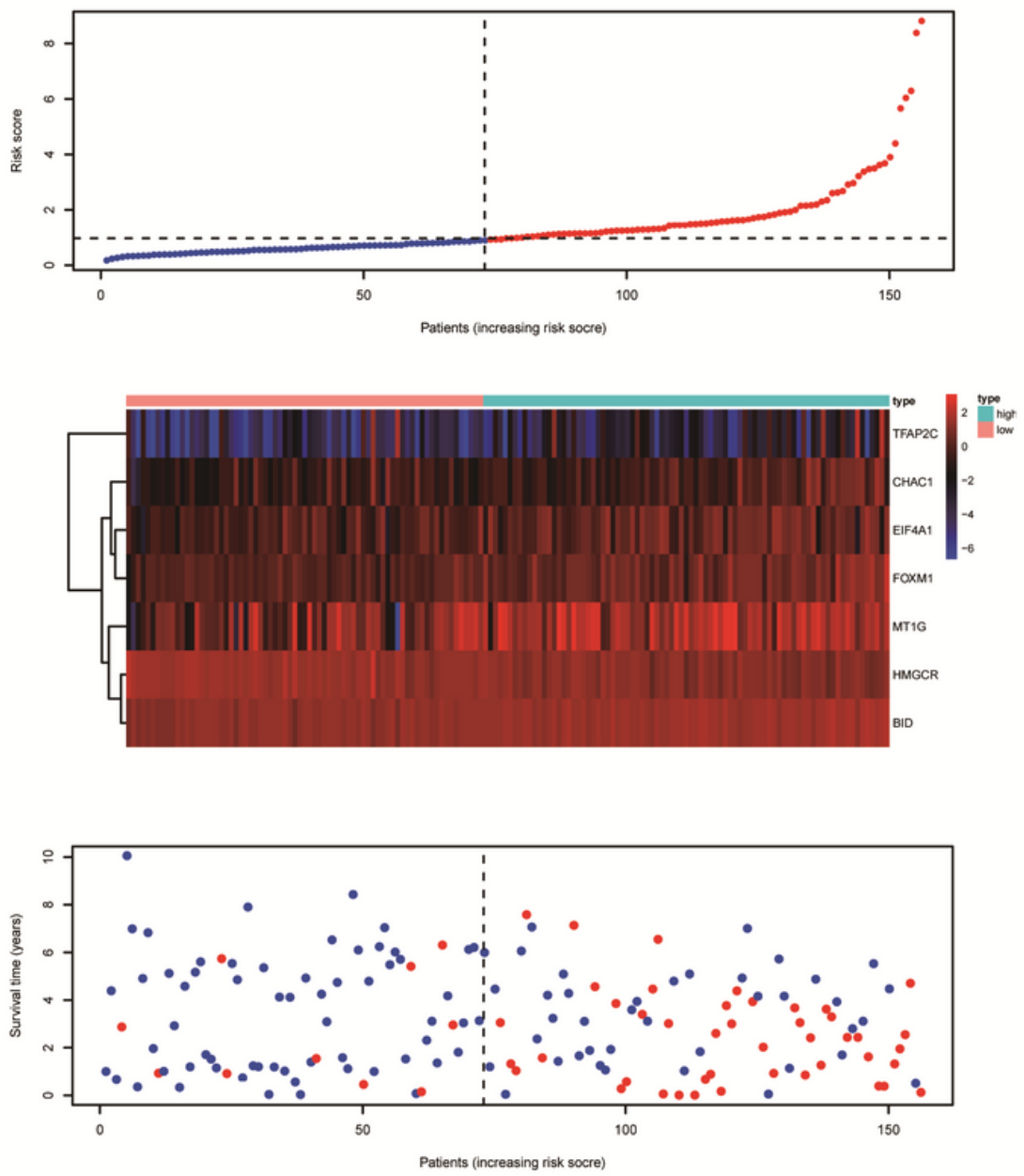

Figure 6

Validation of the prognostic signature based on seven ferroptosis-related genes (FRGs) in the TCGA testing cohort. (A) The Kaplan-Meier survival curves of the 7-FRG prognostic signature; (B) Relationship among the risk score (upper), the expression profiles of the seven prognostic genes (middle), and survival status of patients in different groups (bottom) in the testing cohort; (C) Time-dependent ROC curve of the 7-FRG prognostic signature in the TCGA testing cohort. ROC, receiver operating characteristic curve; TCGA, The Cancer Genome Atlas. 
A

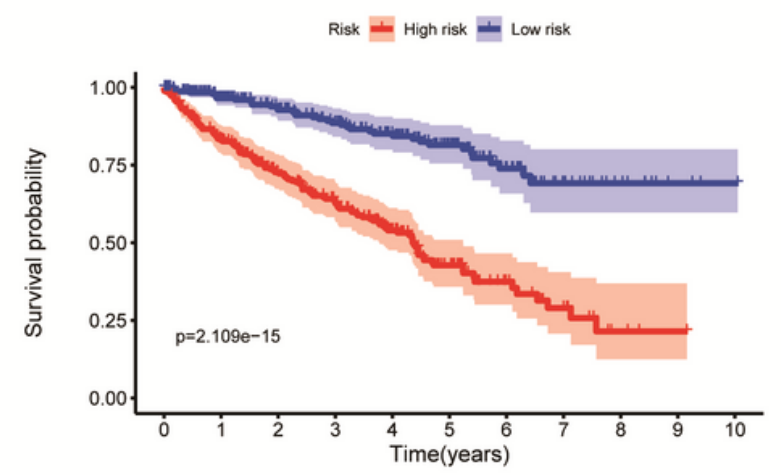

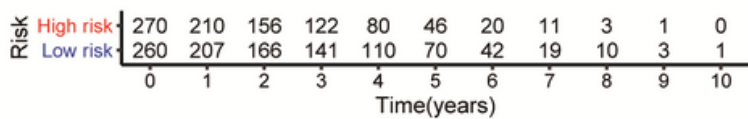

C

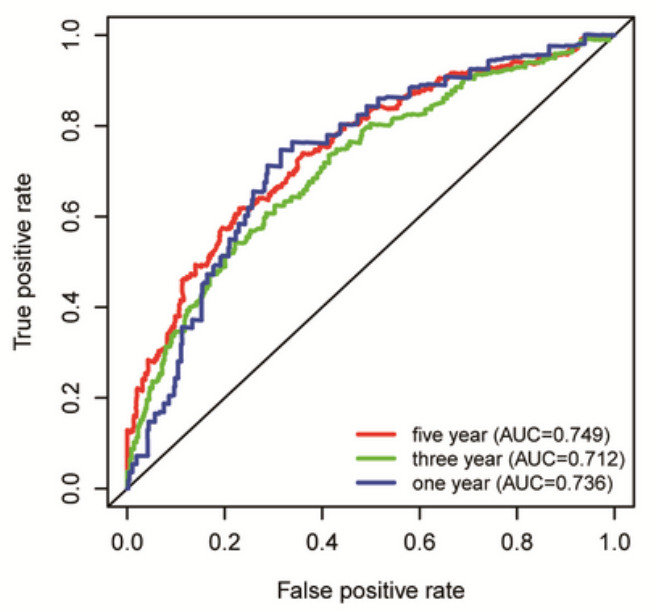

B
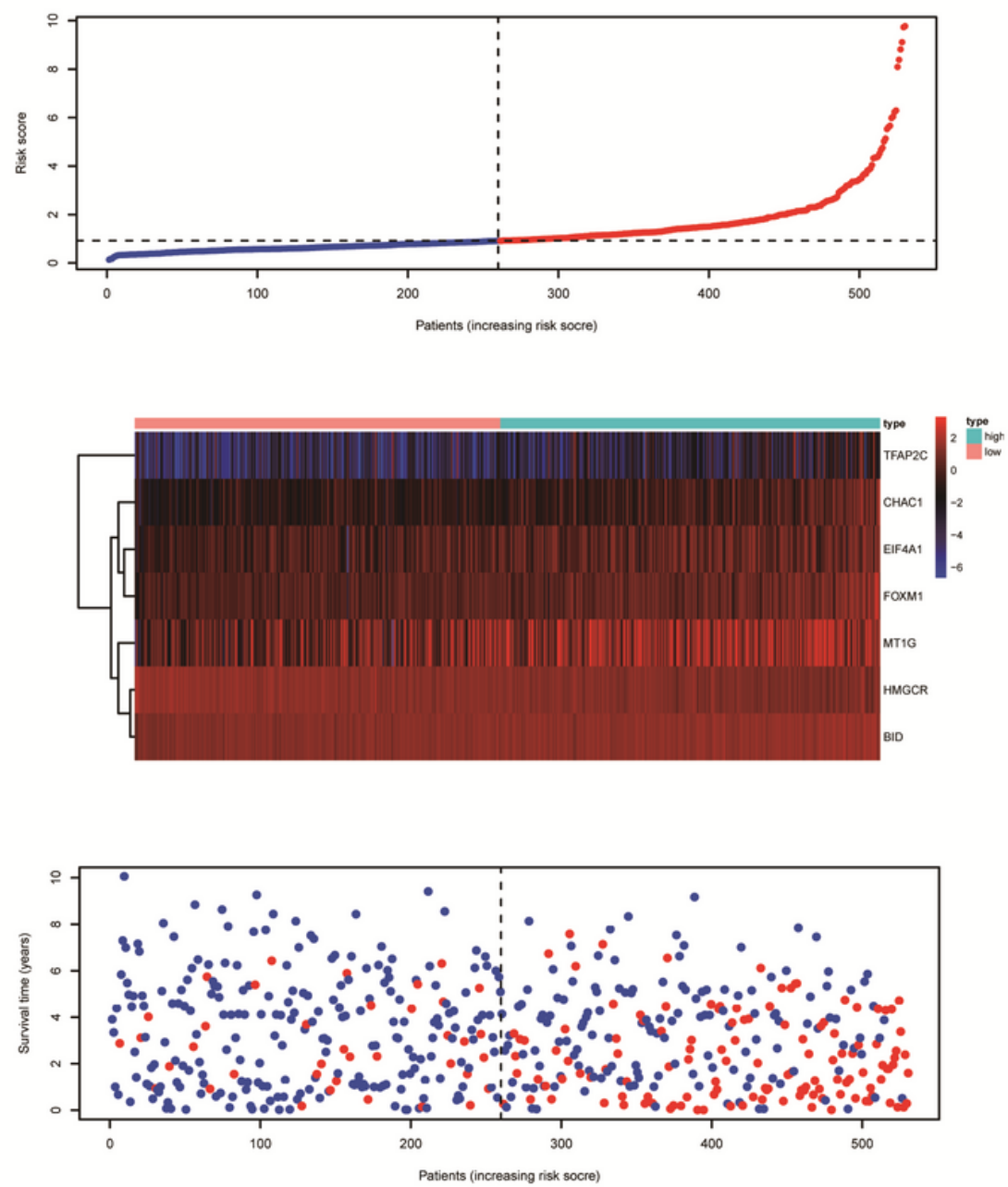

Figure 7

Validation of the prognostic signature based on seven ferroptosis-related genes (FRGs) in the entire TCGA-KIRC dataset. (A) The Kaplan-Meier survival curves of the 7-FRG prognostic signature; (B) Relationship among the risk score (upper), the expression profiles of the seven prognostic genes (middle), and survival status of patients in different groups (bottom) in the entire TCGA-KIRC dataset; (C) Timedependent ROC curve of the 7-FRG prognostic signature in the entire TCGA-KIRC dataset. ROC, receiver operating characteristic curve; TCGA, The Cancer Genome Atlas; KIRC, Kidney renal clear cell carcinoma. 
A

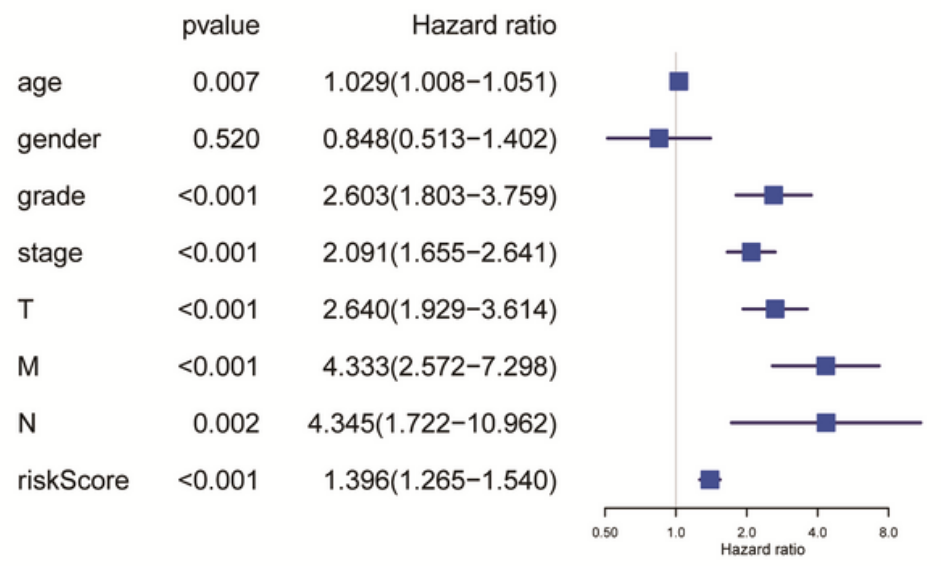

B

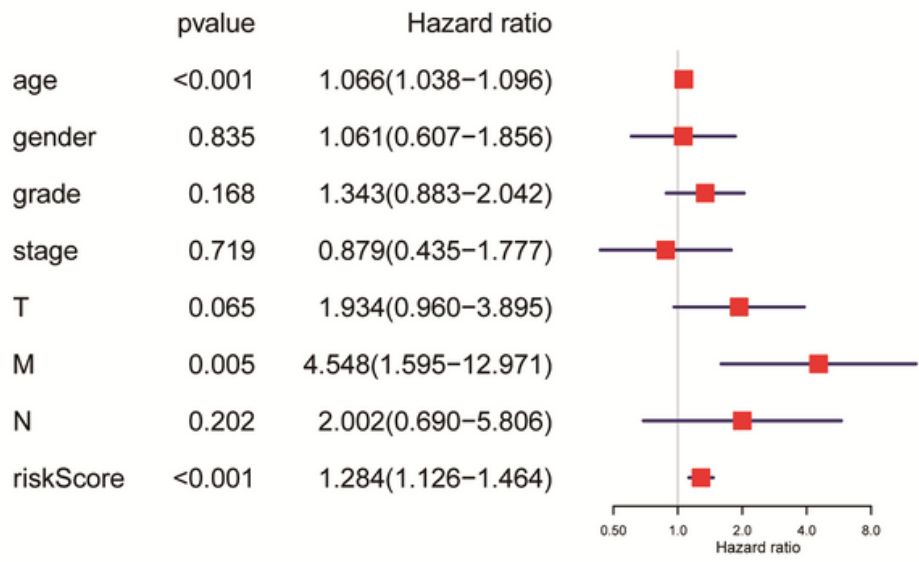

\section{Figure 8}

Univariate analysis and multivariate analysis of risk factors for prognosis in TCGA-KIRC training cohort. (A) Univariate Cox regression analysis; (B) Multivariate Cox regression analysis. TCGA, The Cancer Genome Atlas; KIRC, Kidney renal clear cell carcinoma. 


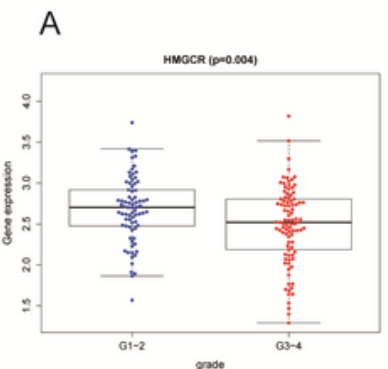

E
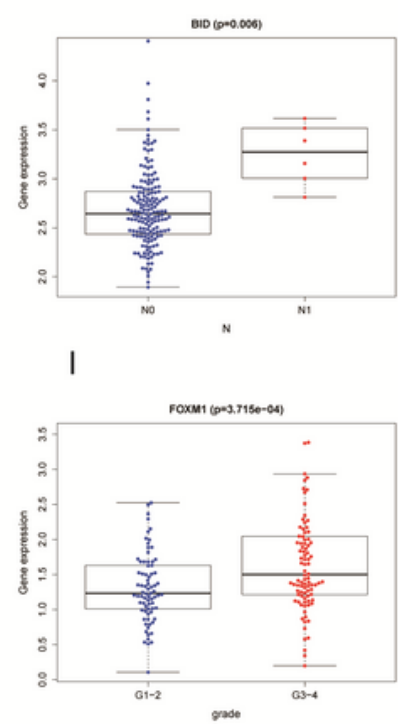

M

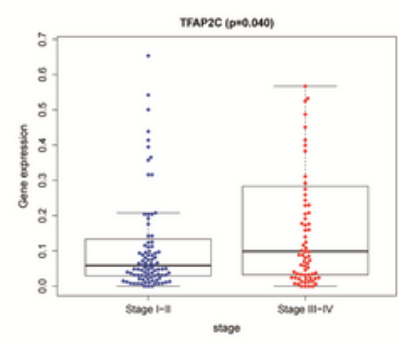

Q

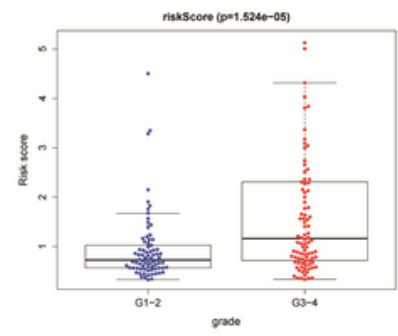

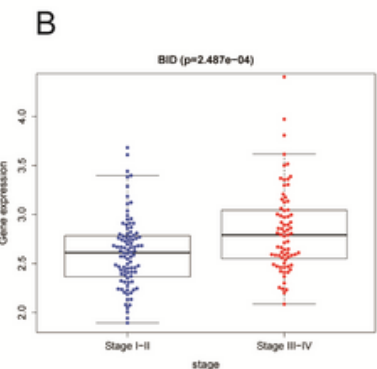

F

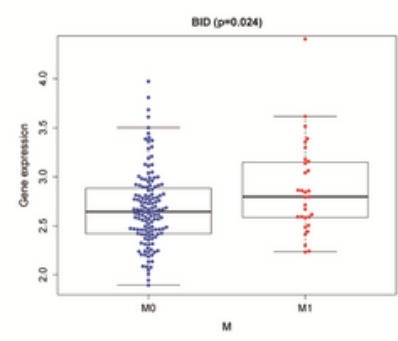

J

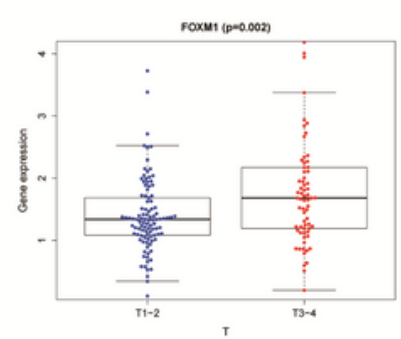

N

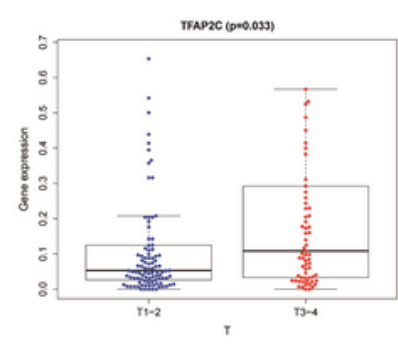

R

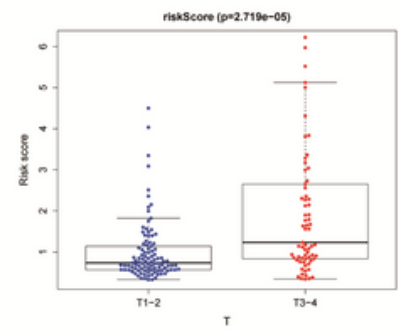

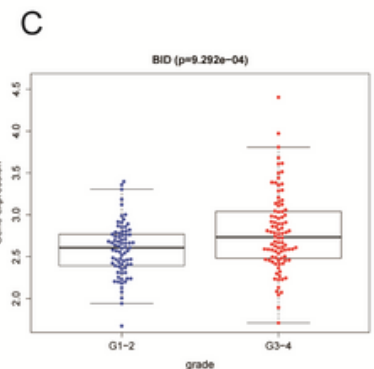

G

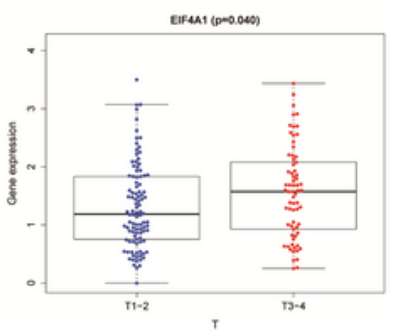

K

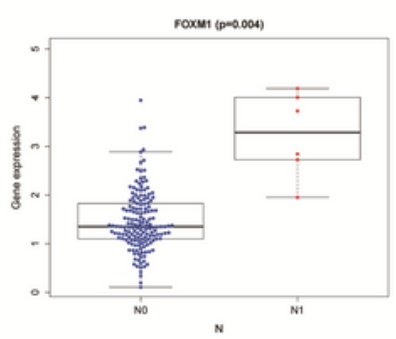

o

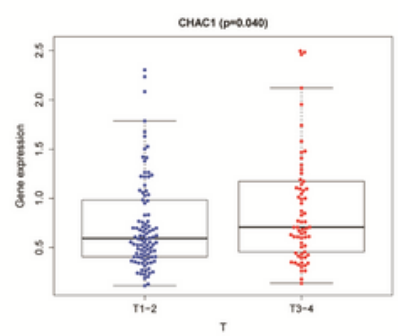

S

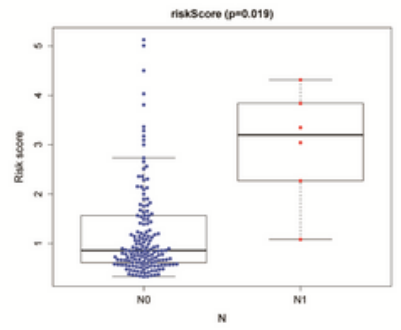

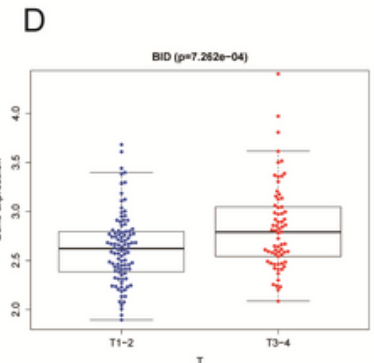

$\mathrm{H}$

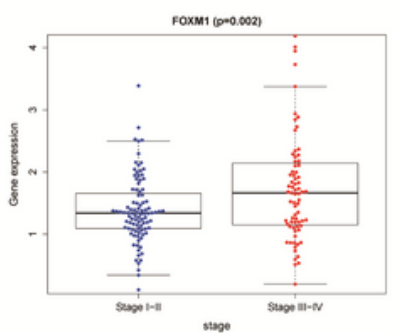

L

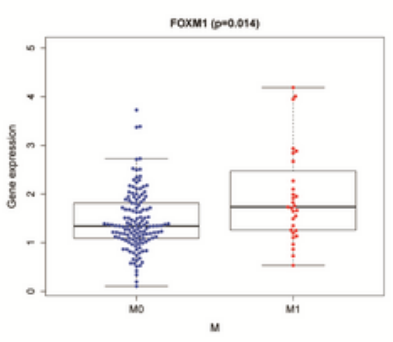

P

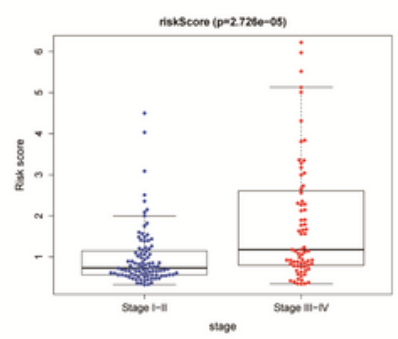

T

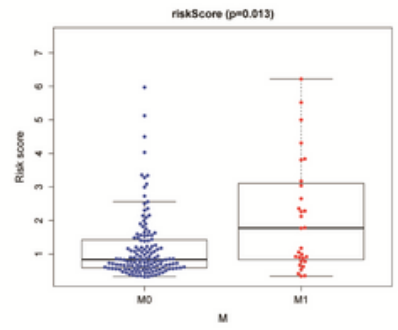

\section{Figure 9}

The relationship between seven hub FRGs and clinical parameters in the TCGA-KIRC training cohort patients. FRGs, ferroptosis-related genes; KIRC, Kidney renal clear cell carcinoma. 
A

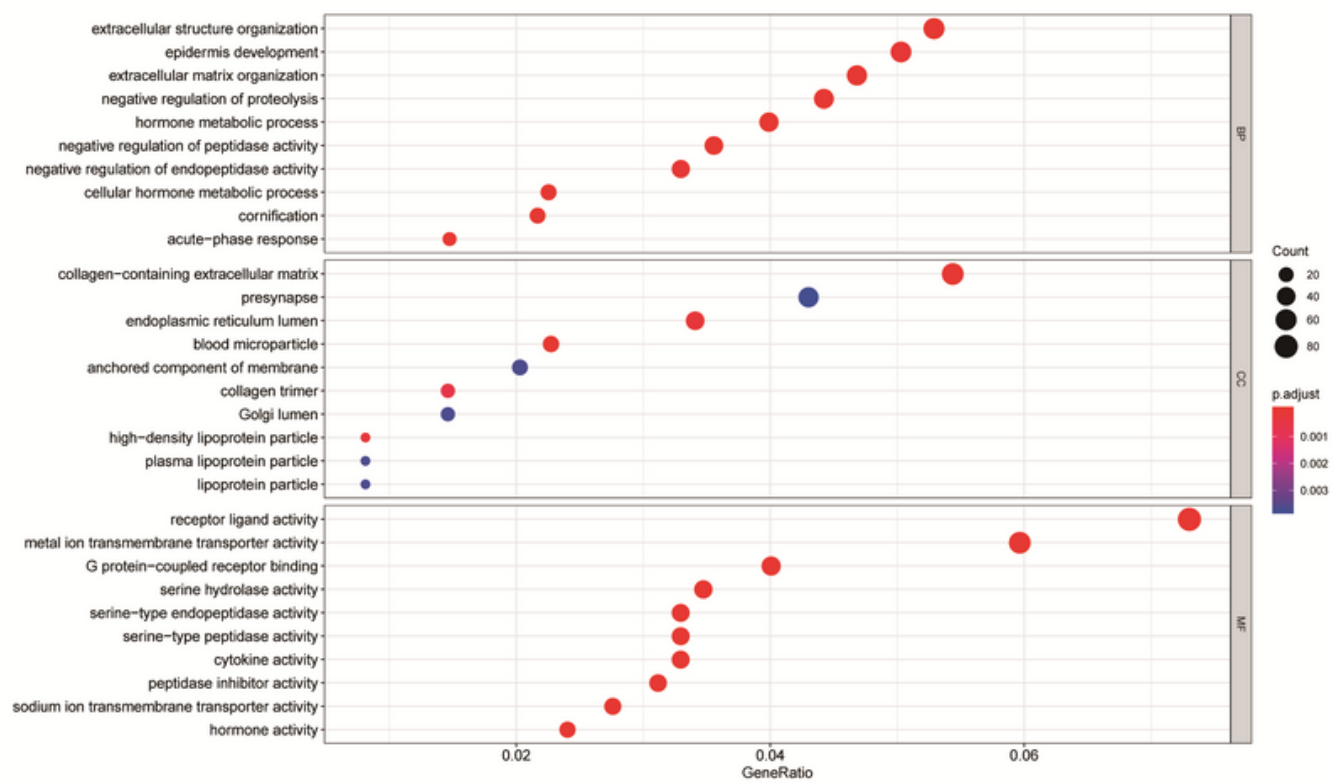

B

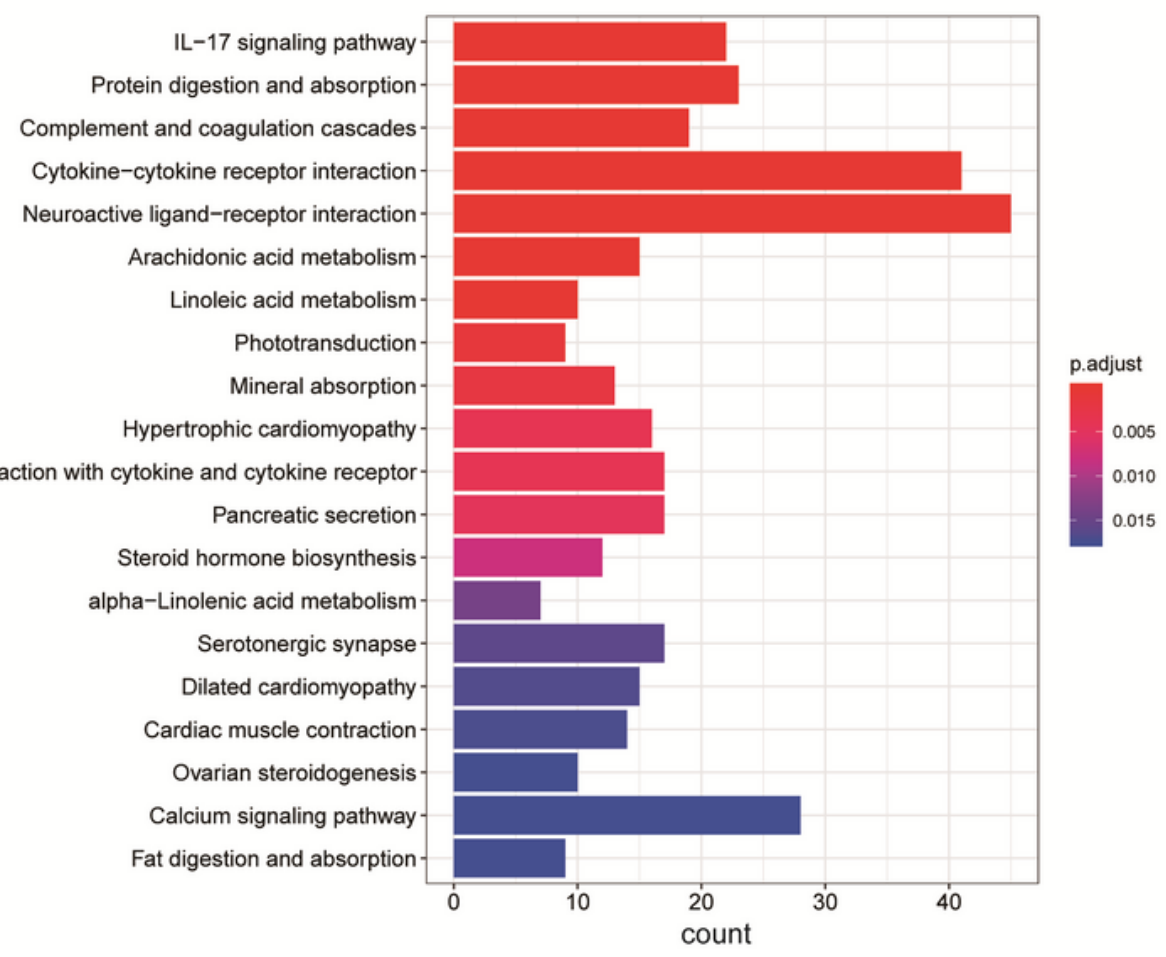

Figure 10

Functional analysis of differentially expressed genes between high and low risk groups. (A) Significantly enriched GO terms; (B) KEGG signaling pathway analysis. GO, Gene Ontology; BP, biological process; CC, cellular component; MF, molecular function; KEGG, Kyoto Encyclopedia of Genes and Genomes pathways. 
A

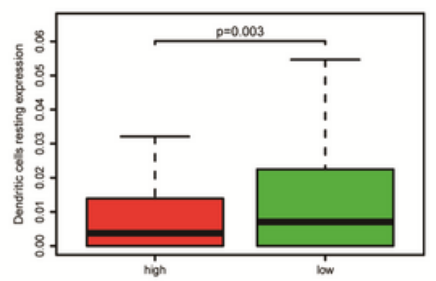

E

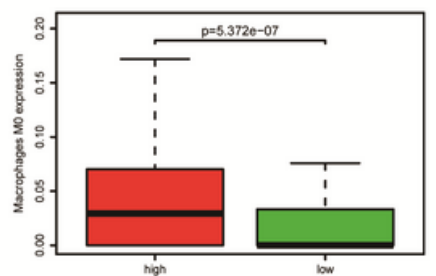

B

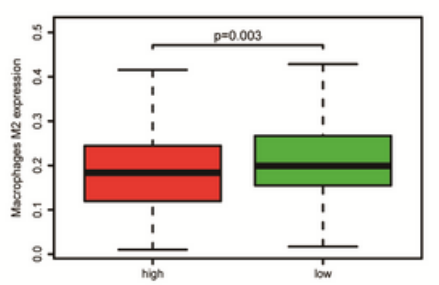

F

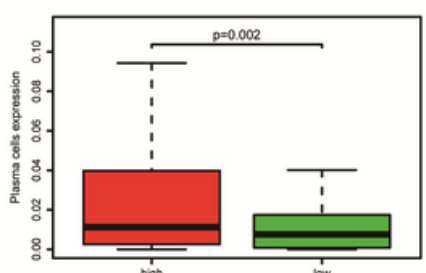

C

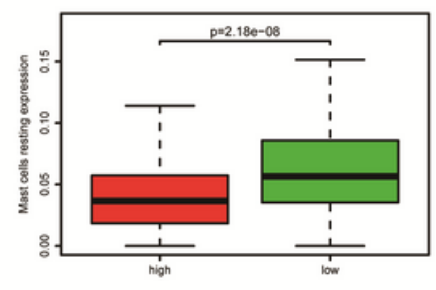

G

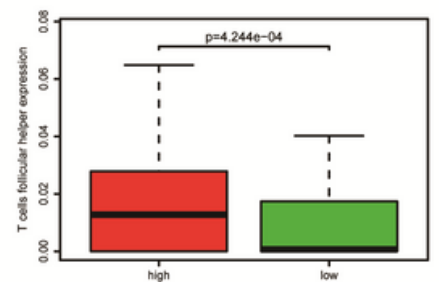

D
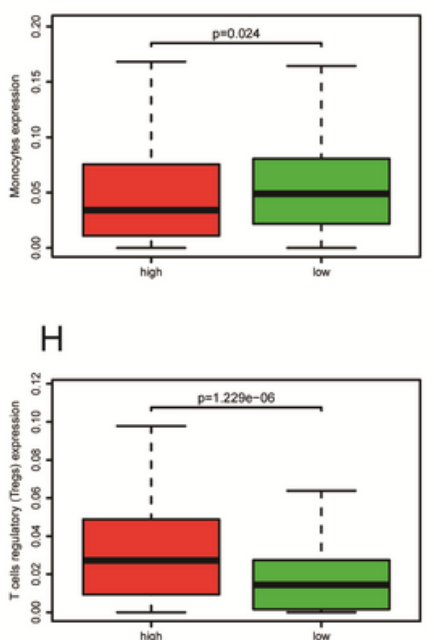

Figure 11

Differences in tumor immune infiltrating cell abundances between high and low risk groups. (A-D) Dendritic cells resting, Macrophages M2, Mast cells resting and Monocytes were significantly enriched in the low-risk group; (E-H) Macrophages M0, Plasma cells, T cells follicular helper and T cells regulatory (Tregs) enriched more in the high-risk group.

A

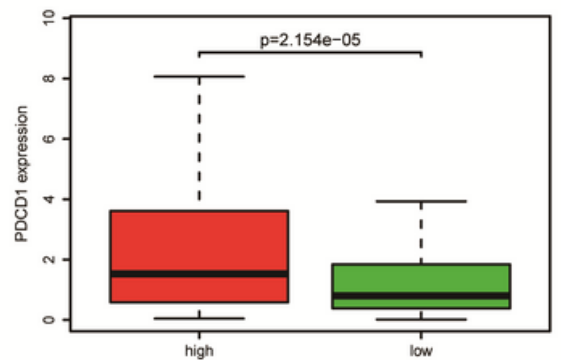

C

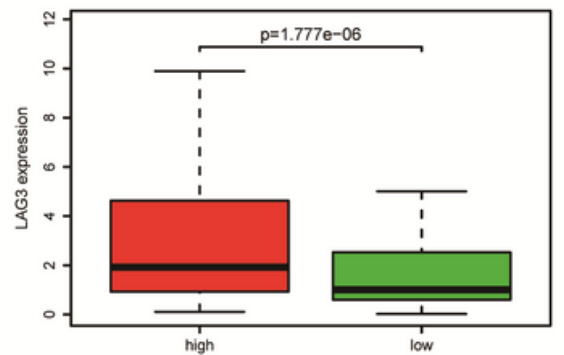

B

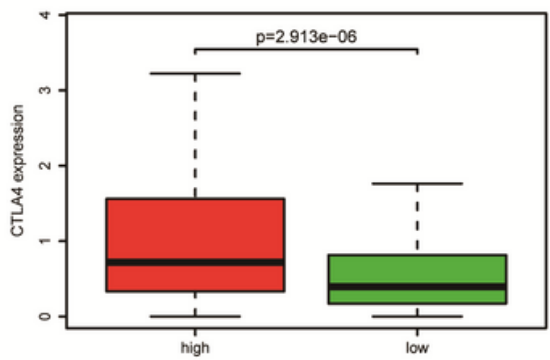

E
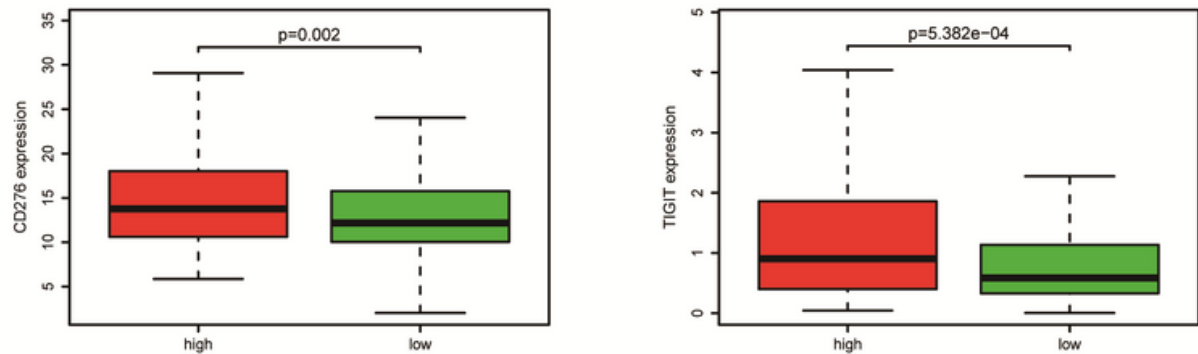

Figure 12

Differences in immune checkpoint expression between high and low risk groups (A) PDCD1; (B) CTLA4; (C) LAG3; (D); (E) CD276; (F) TIGIT. 

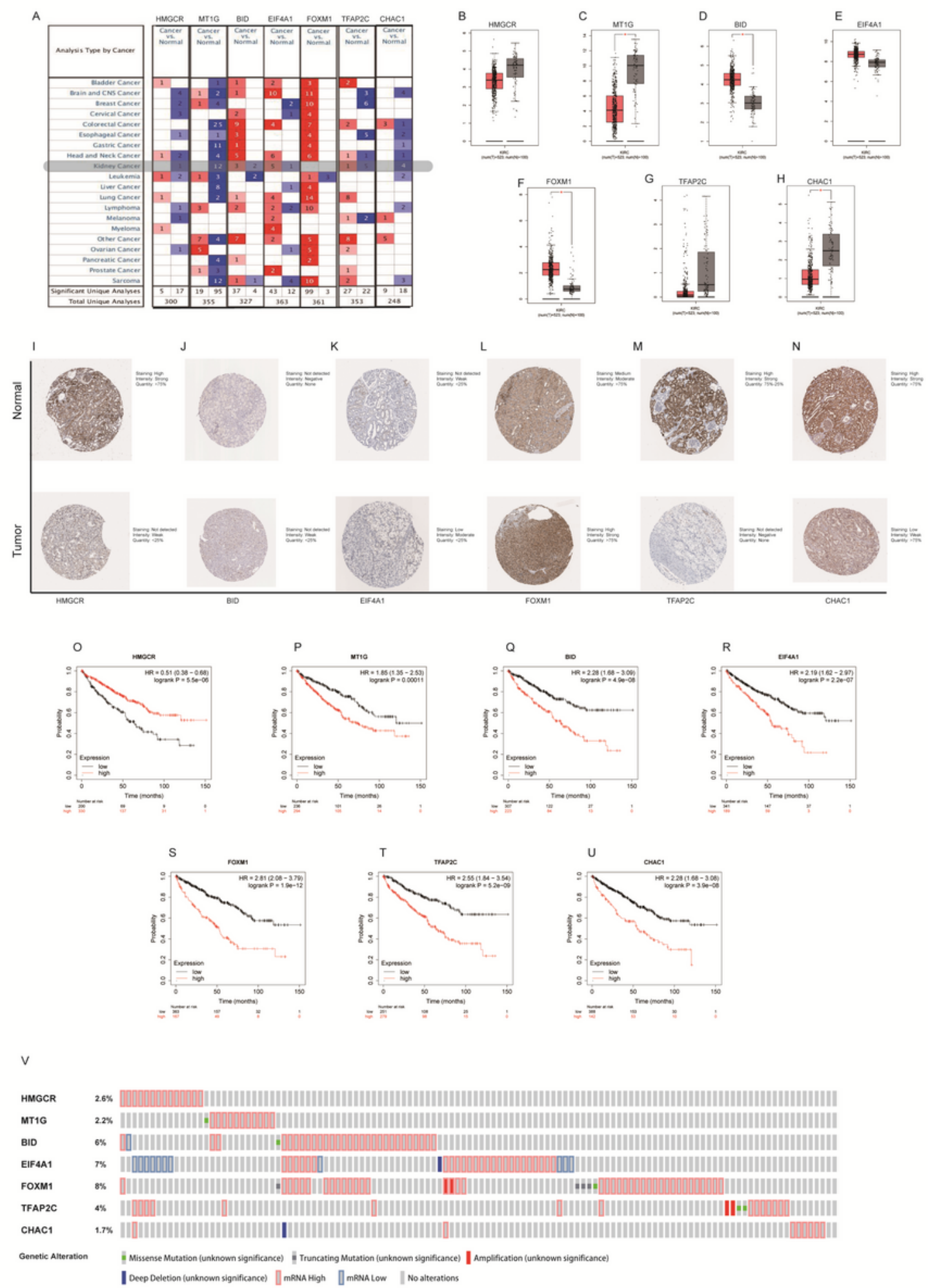

Figure 13

External validation of seven hub FRGs. (A) Expression levels of hub FRGs in different types of human cancers (ONCOMINE database); (B-H) Transcription levels of hub FRGs in KIRC (GEPIA database); (I-N) Immunohistochemistry analysis for hub FRGs in ccRCC (HPA database); (O-U) Kaplan-Meier survival curves of seven hub FRGs (Kaplan-Meier plotter database); (V) Mutation Analysis of hub FRGs in KIRC 
(cBioPortal database). FRGs, ferroptosis-related genes; ccRCC, clear cell renal cell carcinoma; KIRC, Kidney renal clear cell carcinoma.

\section{Supplementary Files}

This is a list of supplementary files associated with this preprint. Click to download.

- Tables1.docx

- TableS2.docx 